\title{
Near-intrinsic energy resolution for 30-662 keV gamma rays in a high pressure xenon electroluminescent TPC
}

V. Álvarez a , F.I.G.M. Borges ${ }^{b}$, S. Cárcel ${ }^{a}, J$ Castel $^{c}$, S. Cebrián ${ }^{c}$, A. Cervera ${ }^{a}$, C.A.N. Conde ${ }^{b}$, T. Dafni $^{c}$, T.H.V.T. Dias ${ }^{\text {b }}$, J. Díaz ${ }^{\text {a }}$, M. Egorov ${ }^{\mathrm{e}}$, R. Esteve ${ }^{\mathrm{d}}$, P. Evtoukhovitch ${ }^{\mathrm{f}}$, L.M.P. Fernandes ${ }^{\mathrm{b}}$, P. Ferrario ${ }^{\mathrm{a}}$, A.L. Ferreira ${ }^{\text {l, E.D.C. Freitas }}{ }^{\text {b }}$, V.M. Gehman ${ }^{\mathrm{e}}$, A. Gil ${ }^{\mathrm{a}}$, A. Goldschmidt ${ }^{\mathrm{e}, *}$, H. Gómez $^{\mathrm{c}}$, J.J. Gómez-Cadenas a , D. González-Díaz ${ }^{c}$, R.M. Gutiérrez ${ }^{\text {h }}$, J. Hauptman ${ }^{i}$, J.A. Hernando Morata ${ }^{j}$, D.C. Herrera ${ }^{c}$, F.J. Iguaz ${ }^{\text {c }}$, I.G. Irastorza ${ }^{c}$, M.A. Jinete ${ }^{\mathrm{h}}$, L. Labarga ${ }^{\mathrm{k}}$, I. Liubarsky ${ }^{\mathrm{a}}$, J.A.M. Lopes ${ }^{\mathrm{b}}$, D. $^{\text {Lorca }}{ }^{\mathrm{a}}$,

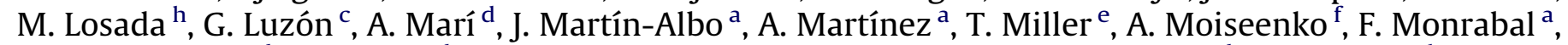
C.M.B. Monteiro $^{\text {b }}$, F.J. Mora ${ }^{\text {d, L.M. Moutinho }}{ }^{g}$, J. Muñoz Vidal ${ }^{\text {a }}$, H. Natal da Luz ${ }^{b}$, G. Navarro ${ }^{\text {, }}$, M. Nebot-Guinot ${ }^{a}$, D. Nygren ${ }^{\mathrm{e}}$, C.A.B. Oliveira ${ }^{\mathrm{e}}$, R. Palma ${ }^{1}$, J. Pérez ${ }^{\mathrm{m}}$, J.L. Pérez Aparicio ${ }^{1}$, J. Renner ${ }^{\mathrm{e}}$, L. Ripoll $^{\text {n }}$, A. Rodríguez ${ }^{\text {, }}$ J. Rodríguez ${ }^{\text {a }}$, F.P. Santos ${ }^{\text {b }}$, J.M.F. dos Santos ${ }^{\text {b }}$, L. Segui ${ }^{c}$, L. Serra $^{\text {a }}$, D. Shuman ${ }^{\mathrm{e}}$, A. Simón ${ }^{a}$, C. Sofka ${ }^{o}$, M. Sorel ${ }^{a}$, J.F. Toledo ${ }^{d}$, A. Tomás ${ }^{c}, J_{\text {. Torrent }}{ }^{n}$, Z. Tsamalaidze ${ }^{\text {f }}$, D. Vázquez $^{\mathrm{j}}$, J.F.C.A. Veloso ${ }^{\text {g }}$, J.A. Villar ${ }^{c}$, R.C. Webb ${ }^{\text {, }}$, J.T. White ${ }^{\mathrm{o}}$, N. Yahlali ${ }^{\text {a }}$

${ }^{a}$ Instituto de Física Corpuscular (IFIC), CSIC \& Universitat de València, Calle Catedrático José Beltrán, 2, 46980 Paterna, Valencia, Spain

${ }^{\mathrm{b}}$ Departamento de Fisica, Universidade de Coimbra, Rua Larga, 3004-516 Coimbra, Portugal

${ }^{\mathrm{c}}$ Laboratorio de Física Nuclear y Astropartículas, Universidad de Zaragoza, Calle Pedro Cerbuna 12, 50009 Zaragoza, Spain

${ }^{\mathrm{d}}$ Instituto de Instrumentación para Imagen Molecular (I3M), Universitat Politècnica de València, Camino de Vera, s/n, Edificio 8B, 46022 Valencia, Spain

e Lawrence Berkeley National Laboratory (LBNL), 1 Cyclotron Road, Berkeley, California 94720, USA

f Joint Institute for Nuclear Research (JINR), Joliot-Curie 6, 141980 Dubna, Russia

${ }^{\mathrm{g}}$ Institute of Nanostructures, Nanomodelling and Nanofabrication (i3N), Universidade de Aveiro, Campus de Santiago, $3810-193$ Aveiro, Portugal

${ }^{\mathrm{h}}$ Centro de Investigaciones en Ciencias Básicas y Aplicadas, Universidad Antonio Nariño, Carretera 3 este No. 47A-15, Bogotá, Colombia

i Department of Physics and Astronomy, Iowa State University, 12 Physics Hall, Ames, Iowa 50011-3160, USA

${ }^{\mathrm{j}}$ Instituto Gallego de Física de Altas Energías (IGFAE), Univ. de Santiago de Compostela, Campus sur, Rúa Xosé María Suárez Núñez, s/n, 15782 Santiago de Compostela, Spain

${ }^{k}$ Departamento de Física Teórica, Universidad Autónoma de Madrid, Campus de Cantoblanco, 28049 Madrid, Spain

${ }^{1}$ Dpto. de Mecánica de Medios Continuos y Teoría de Estructuras, Univ. Politècnica de València, Camino de Vera, s/n, 46071 Valencia, Spain

m Instituto de Física Teórica (IFT), UAM/CSIC, Campus de Cantoblanco, 28049 Madrid, Spain

${ }^{n}$ Escola Politècnica Superior, Universitat de Girona, Av. Montilivi, s/n, 17071 Girona, Spain

o Department of Physics and Astronomy, Texas A\&'M University, College Station, Texas 77843-4242, USA

\section{A R T I C L E I N F O}

\section{Article history:}

Received 21 December 2012

Accepted 31 December 2012

Available online 18 January 2013

\section{Keywords:}

Xenon

Energy resolution

High-pressure

TPC

Electroluminescence

Neutrinoless double beta decay

\begin{abstract}
A B S T R A C T
We present the design, data and results from the NEXT prototype for Double Beta and Dark Matter (NEXT-DBDM) detector, a high-pressure gaseous natural xenon electroluminescent time projection chamber (TPC) that was built at the Lawrence Berkeley National Laboratory. It is a prototype of the planned NEXT- $100{ }^{136}$ Xe neutrino-less double beta decay $(0 v \beta \beta)$ experiment with the main objectives of demonstrating near-intrinsic energy resolution at energies up to $662 \mathrm{keV}$ and of optimizing the NEXT-100 detector design and operating parameters. Energy resolutions of $\sim 1 \%$ FWHM for $662 \mathrm{keV}$ gamma rays were obtained at 10 and $15 \mathrm{~atm}$ and $\sim 5 \%$ FWHM for $30 \mathrm{keV}$ fluorescence xenon X-rays. These results demonstrate that 0.5\% FWHM resolutions for the $2459 \mathrm{keV}$ hypothetical neutrino-less double beta decay peak are realizable. This energy resolution is a factor 7-20 better than that of the current leading $0 v \beta \beta$ experiments using liquid xenon and thus represents a significant advancement. We present also first results from a track imaging system consisting of 64 silicon photo-multipliers recently installed in NEXT-DBDM that, along with the excellent energy resolution, demonstrates the key functionalities required for the NEXT-100 $0 v \beta \beta$ search.
\end{abstract}

(c) 2013 Elsevier B.V. All rights reserved.

\footnotetext{
${ }^{*}$ Corresponding author. Tel.: +1 5104867518 .

E-mail address: agoldschmidt@lbl.gov (A. Goldschmidt).
} 


\section{Introduction}

Neutrino-less double beta decay $(0 v \beta \beta)$ is a postulated [1] rare process in which a nucleus changes by two units of charge while emitting two electrons (or positrons) without the emission of neutrinos [2]. Should this decay happen in nature the sum of the energies of the two electrons will be monoenergetic at exactly the $Q$-value of the nuclear decay $\left(Q_{\beta \beta}\right.$, equal to the mass difference between the parent and daughter nuclei). A precise energy measurement can therefore greatly aid in the identification of the $0 v \beta \beta$ process in the presence of other more common processes that produce either continuous energy deposition spectra or peaks at well known and well separated energies. The occurrence of $0 v \beta \beta$ would imply that neutrinos are their own antiparticle $[3,4]$, i.e. Majorana particles. Should neutrinos prove to be Majorana particles, the observed prevalence of matter over antimatter in our universe could be explained through the Leptogenesis mechanism (see e.g. [5,6]).

The ${ }^{136} \mathrm{Xe}$ isotope is one of the candidate nuclei in which $0 v \beta \beta$, in this case ${ }^{136} \mathrm{Xe} \rightarrow{ }^{136} \mathrm{Ba}+e^{-}+e^{-}$, could be detectable because the single beta decay which would otherwise dominate the experimental count rate is energetically forbidden. Two currently running experiments are searching for this rare process in ${ }^{136} \mathrm{Xe}$. EXO [7] uses $200 \mathrm{~kg}$ of the enriched isotope in a cryogenic liquid xenon TPC and KamLAND-Zen [8] uses $330 \mathrm{~kg}$ of the isotope dissolved in 13 tons of organic scintillator. The energy resolutions for these two experiments are 3\%-4\% and 10\% FWHM respectively at the $2.459 \mathrm{MeV}[9] Q_{\beta \beta}$ of the ${ }^{136} \mathrm{Xe}$ decay. It is well known, on the other hand, that xenon in gaseous phase can offer significantly better energy resolution due to its small Fano factor [10] $F=0.14$ (a measure of the level of fluctuations in the number of ionization electrons for a fixed energy deposition). For a xenon gas pressure of less than $57 \mathrm{~atm}$ at room temperature $\left(0.55 \mathrm{~g} / \mathrm{cm}^{3}\right.$ density $)$ the intrinsic energy resolution is expected to be Ref. [11] about $0.3 \%$ FWHM near the $Q_{\beta \beta}$. It is thus clear that a xenon detector at moderately high pressure would represent a significant advantage for the search of the $0 v \beta \beta$ spectrum peak as long as its implementation can preserve a near-intrinsic energy resolution.

NEXT-100 is an experiment [12] being constructed to search for $0 v \beta \beta$ using $100-150 \mathrm{~kg}$ of ${ }^{136} \mathrm{Xe}$ in a $10-15 \mathrm{~atm}$ TPC at the Canfranc Underground Laboratory (LSC) under the Pyrenees mountains in Spain. In the TPC, conceptually developed in Ref. [13], electrons liberated through ionization by the passage of energetic charged particles (such as the two electrons from the $0 v \beta \beta$ decay) drift under the presence of a weak electric field towards a thin region with a high electric field. The $E / P$ (electric field divided by pressure) in this high field region is such that electrons acquire enough energy to excite xenon atoms, but not enough to ionize them. Most of the excitation energy is ultimately released in the form of ultra-violet (VUV) photons of wavelengths near $172 \mathrm{~nm}$ and constitutes the electroluminescence (EL) [14] signal. For each ionization electron, thousands of EL photons can be produced in the EL amplification region, with extremely low fluctuations in the number of EL photons produced. An array of photomultiplier tubes (PMTs) then detects a fraction of the VUV photons to render a measurement of the total energy released in the gas with a statistical precision near the Fano limit.

The NEXT-100 TPC will provide, in addition to a very precise energy measurement, a 3-D image of the ionization tracks by means of a dense array of silicon photomultipliers [15] (SiPMs or MPPCs) installed near the electroluminescence region. This topological information is useful to distinguish between events with 2 electrons emerging from a single point, such as in $0 v \beta \beta$, from events with one or more electrons that result from single-site and multi-site interactions of gamma rays from natural radioactivity in the detector and surrounding materials.
In this paper we present the design, data and results from the NEXT-DBDM (NEXT prototype for Double Beta and Dark Matter) detector, a $1 \mathrm{~kg}$ natural xenon electroluminescent TPC that was built at the Lawrence Berkeley National Laboratory. It is a prototype of the NEXT-100 detector with the main objectives of demonstrating the near intrinsic energy resolution at energies up to $662 \mathrm{keV}$ and of optimizing the NEXT-100 detector design, construction, and operating parameters.

\section{High pressure xenon electroluminescent TPC}

In the past, various high pressure xenon detector designs have been created for studies directed towards the improvement of energy resolution. Studies of gridded ionization chambers in cylindrical form [16] and with parallel plate geometries [17-19,11] were able to achieve energy resolutions at the level of $2 \%-2.5 \%$ FWHM at $662 \mathrm{keV}$. Later studies investigated alternatives to shielding grids such as a hemispherical geometry [20], using a virtual Frisch grid [21], pulse correction using drift time determined by observing primary scintillation [22], and variations [21,23] of the coplanar anode approach introduced in Ref. [24]. These studies produced energy resolutions of $2 \%-6 \%$ FWHM at $662 \mathrm{keV}$. It has been shown in Ref. [25] that good energy resolution (2.7\% FWHM at $122 \mathrm{keV}$ ) is achievable using electroluminescent readout (see Ref. [13] for a more thorough historical account of electroluminescence in detectors), and here we extend the usage of this technique to higher energies.

The basic building blocks of the NEXT-DBDM xenon electroluminescent TPC are: a stainless steel pressure vessel, a gas system that recirculates and purifies the xenon at $10-15 \mathrm{~atm}$, stainless steel wire meshes that establish high-voltage equipotential planes in the boundaries of the drift and the EL regions, field cages with hexagonal cross-sections to establish uniform electric fields in those regions, an hexagonal pattern array of 19 VUV sensitive PMTs inside the pressure vessel and an associated readout electronics and data acquisition (DAQ) system.

When ionizing radiation traverses the drift region of the TPC, xenon atoms are ionized or excited. Most of the excitation energy is promptly released as a fast scintillation pulse of $172 \mathrm{~nm}$ VUV photons that lasts 10-30 ns [14]. A fraction of these photons are detected in the PMT array, forming the $\mathrm{S} 1$ signal that provides the start time $t_{0}$ for the TPC. The ionization (or secondary) electrons, on the other hand, drift at a velocity of $\sim 0.1 \mathrm{~cm} / \mu$ s towards the EL region. There, they accelerate and produce copious EL VUV photons. The same PMT array detects a fraction of these photons, forming the S2 signal.

In the NEXT-DBDM detector the PMT array and the EL region, which are both hexagonal areas with $12.4 \mathrm{~cm}$ between opposite sides, are $13.5 \mathrm{~cm}$ away from each other (see Fig. 1). Thus pointlike isotropic light produced in the EL region illuminates the PMT array with little PMT-to-PMT variation. This geometric configuration also makes the illumination pattern and the total light collection only very mildly dependent on the position of the light origin within the EL region. The diffuse reflectivity of the TPC walls increases this light collection uniformity further. As a result, the device provides good energy measurements with little dependence on the position of the charge depositions. On the other hand, without a light sensor array near the EL region precise tracking information is not available and only coarse average position can be obtained using the PMT array light pattern. In this report, only the tracking analysis in Section 8.1 was performed using data acquired with the tracking sensor array recently installed in the NEXT-DBDM TPC. In a future publication we will report on the energy resolution achieved in a combined analysis using information from both the PMT and tracking arrays. 


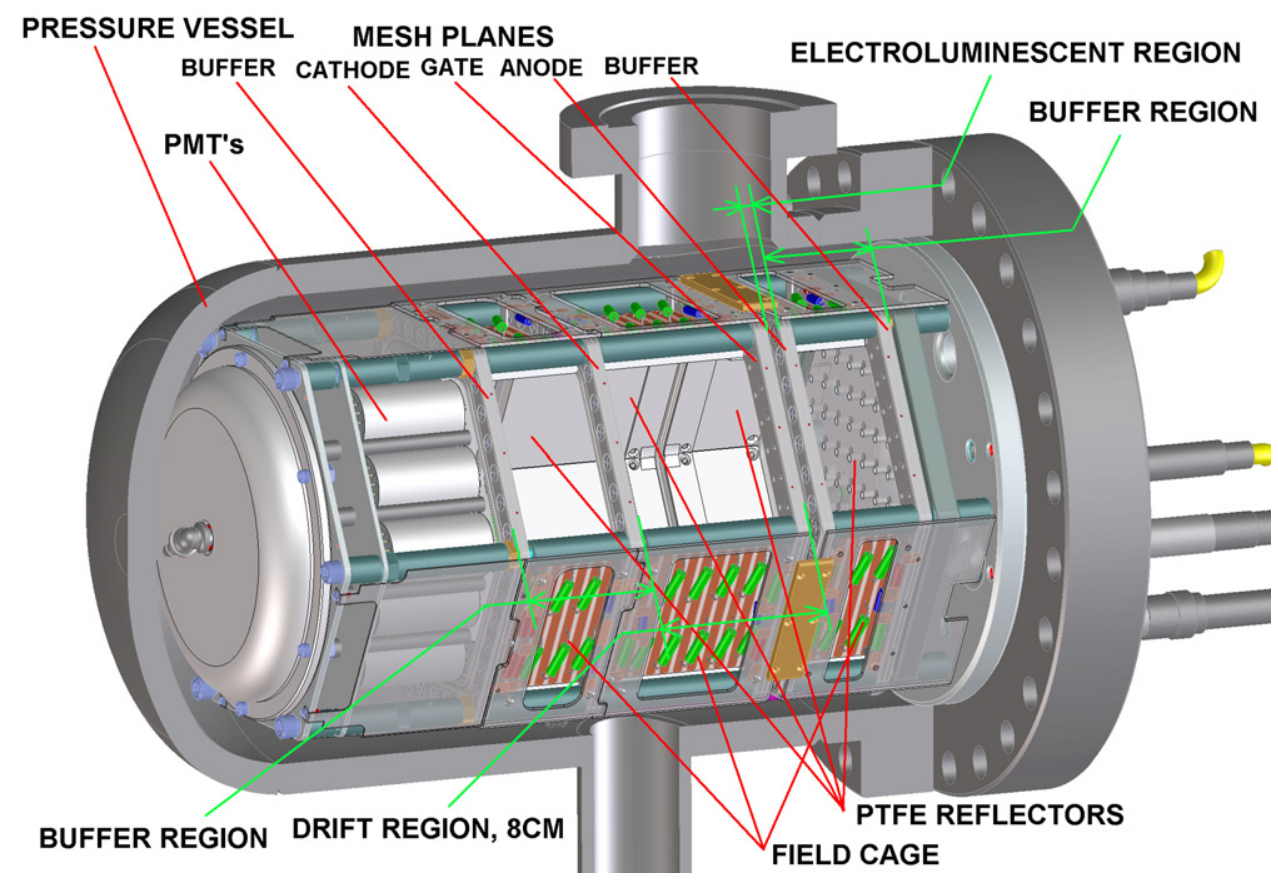

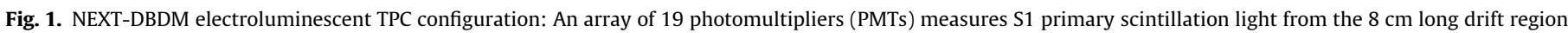

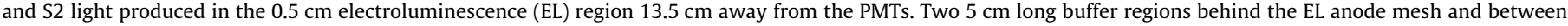
the PMTs and the cathode mesh grade the high voltages (up to $\pm 17 \mathrm{kV}$ ) down to ground potential.

\section{Intrinsic energy resolution in the HPXe TPC}

The intrinsic energy resolution in a xenon gas detector that measures ionization is given by

$\delta E / E=2.35 \sqrt{F W_{\mathrm{i}} / E} \quad(\mathrm{FWHM})$

where $E$ is the energy released in the detector, $W_{\mathrm{i}}$ is the average energy required to liberate an electron and $F$ is the Fano factor that quantifies the fluctuations in the number of liberated electrons. $F$ and $W_{\mathrm{i}}$ are energy, drift field and pressure dependent due to electron-ion recombination and due to the energy dependence of the energy loss rate $d E / d x$ (see for example Refs. $[11,26,27])$. In this study we explored the $30-662 \mathrm{keV}$ energy range at pressures between 10 and $17 \mathrm{~atm}$, and drift fields in the $0.3-2.0 \mathrm{kV} / \mathrm{cm}$ range. For the purpose of studying the energy resolution in the NEXT-DBDM detector we used $662 \mathrm{keV}$ gamma rays from a ${ }^{137} \mathrm{Cs}$ source and $29.1-34.5 \mathrm{keV}$ xenon X-rays [28] that follow photoelectric interactions of the gamma rays. For $F=0.14$ [29] and $W_{\mathrm{i}}=24.8 \mathrm{eV}[30,13]$ the intrinsic energy resolutions are approximately 0.53\% FWHM for $662 \mathrm{keV}$ and $2.5 \%$ FWHM for $30 \mathrm{keV}$.

\section{Experimental aspects of the energy resolution in the electroluminescent HPXe TPC}

\subsection{Statistical contributions}

In the NEXT-DBDM detector the number of liberated electrons is not measured directly. Rather, those electrons are drifted towards the EL region and then accelerated to produce $\mathrm{O}(1000)$ VUV photons for each electron crossing the EL region of which $\mathrm{O}(10)$ are measured as photoelectrons in the PMT array. This gain and measurement sequence introduces fluctuations beyond the intrinsic Fano limit. In Ref. [31] a formalism was developed to calculate the energy resolution achievable in light of those additional fluctuations

$\delta E / E=2.35 \sqrt{(F+G) W_{\mathrm{i}} / E} \quad(\mathrm{FWHM})$

with

$G=1 / \eta+\left(1+\sigma_{\mathrm{pd}}^{2}\right) / n_{\mathrm{pe}}$

where $\eta$ is the average number of VUV photons produced in the EL region per secondary electron (or optical gain), $n_{\mathrm{pe}}$ is the average number of photons detected (as photoelectrons) per secondary electron and $\sigma_{\mathrm{pd}}^{2}$ is the variance on the charge measured for single photoelectrons. ${ }^{1}$

The electroluminescence optical gain in pure high pressure xenon is given approximately by $[32,13]$

$\eta=140\left(\frac{\Delta V}{p \Delta z}-0.83\right) \cdot p \cdot \Delta z$

where $\Delta V$ is the voltage difference between the mesh planes that form the EL region in kilovolts, $p$ is the pressure in atmospheres and $\Delta z$ is the distance between the electrodes in centimeters. For instance, if $p=10 \mathrm{~atm}, \Delta V=11.3 \mathrm{kV}$ and $\Delta z=0.5 \mathrm{~cm}$ the $\mathrm{EL}$ optical gain is $\eta=1000$.

The value of $n_{\mathrm{pe}}$ is the product of the EL optical gain $\eta$ times the collection efficiency (the probability of a VUV photon generated in the EL region reaching a PMT window) times the PMT quantum efficiency at the corresponding wavelength. For example

${ }^{1}$ A thorough analysis of the statistics of energy resolution gives the formula

$\frac{\delta E}{E}=2.35 \sqrt{\frac{F}{n_{\text {ion }}}+\frac{J_{\mathrm{CP}}-1}{n_{\mathrm{EL}}}+\frac{\sigma_{\mathrm{pd}}^{2}+1}{n_{\mathrm{det}}}} \quad(\mathrm{FWHM})$

where $n_{\text {ion }}=E / W_{\mathrm{i}}$ is the number of electron-ion pairs produced, $n_{\mathrm{EL}}=n_{\mathrm{ion}} \eta$ is the total number of EL photons, and $n_{\text {det }}=\eta \varepsilon n_{\text {ion }}$ is the total number of photoelectrons detected by the PMTs ( $\varepsilon$ is the detection efficiency of EL photons). $J_{\mathrm{CP}}$ is the CondePolicarpo factor which characterizes the fluctuations in EL light production similar to how the Fano factor $F$ characterizes the fluctuation in ionization production. Eq. (2) assumes $J_{\mathrm{CP}}=2$. In most realistic scenarios, however, the second term under the square root can be neglected due to the low EL photon detection probability. 
for a $10 \%$ collection efficiency, a 15\% PMT quantum efficiency at the $172 \mathrm{~nm}$ wavelength of the xenon electroluminescence and $\eta=1000, n_{\mathrm{pe}}=15$. Then, the expected energy resolution for $662 \mathrm{keV}$ gamma rays in the absence of systematic effects is $0.66 \%$ and $3.11 \%$ for $30 \mathrm{keV}$.

\subsection{Systematic effects}

Systematic effects that broaden the energy resolution can be grouped in two categories: position and time dependencies. Position dependencies of the energy response arise, for example, from a non-uniform EL light collection efficiency, a non-uniform EL gain, secondary electron losses due to attachment on electronegative impurities during drift and secondary electrons hitting the TPC walls due to diffusion or due to drift field nonuniformities. Time dependencies of the energy response arise, for example, from time-varying voltages, temperature (and its subsequent effect of gas viscosity, PMT response, etc.), gas flow and purity, PMT response and gas density.

In the NEXT-DBDM TPC systematic dependencies of the energy response as a function of the position along the drift direction ( $z$-axis in our chosen coordinate system) are small due to the high xenon gas purity achieved but still non-negligible. In addition, the $z$ position of charge depositions within the drift region is very precisely measured. The opposite is true in the plane perpendicular to the drift direction $x-y$ : the light collection changes more rapidly as a function of distance from the center axis of the TPC and the $x-y$ position of charge depositions is poorly measured. For this reason, to study the energy resolution achievable with the xenon EL TPC, calibration gamma rays are introduced along the center $z$ axis through a narrow aperture collimator. Still, the actual charge depositions happen over an extended region in $x-y$ (and $z$ ) due to the length of the electron tracks and due to the multi-site depositions from Compton scatters and from xenon $\mathrm{X}$-rays following photoelectric absorption.

\section{Experimental setup}

\subsection{Gas system}

The main functions of the gas system are to recirculate and purify the xenon at pressures up to $17 \mathrm{~atm}$. A magnetically driven seal-less and oil-less pressure rated (95 atm) pump manufactured by Pumpworks Inc. (model 2070) recirculates the xenon at room temperature at 5-15 standard liter per minute (slpm). For the total system volume of approximately $10 \mathrm{l}$ at $10 \mathrm{~atm}$ pressure the pump recirculates one full volume in about $10 \mathrm{~min}$. A pressure rated (18 atm) heated getter from Johnson Matthey (model PureGuard) removes $\mathrm{O}_{2}, \mathrm{H}_{2} \mathrm{O}, \mathrm{N}_{2}$ and many other impurities using a non-evaporable zirconium-based material. The getter operates at a constant $450{ }^{\circ} \mathrm{C}$ irreversibly removing the impurities through bulk diffusion.

In Fig. 2 the complete gas system is shown. Besides the recirculation pump and the heated getter the system includes a vacuum system with a roughing pump (make TriboVacDry), a turbomolecular pump (make Leybold-Heareus) and Pirani and ion vacuum gauges, a reclamation cylinder where xenon is stored after it is cryogenically removed from the main pressure vessel, an argon purge system, a gas sampling system with a precision leak valve

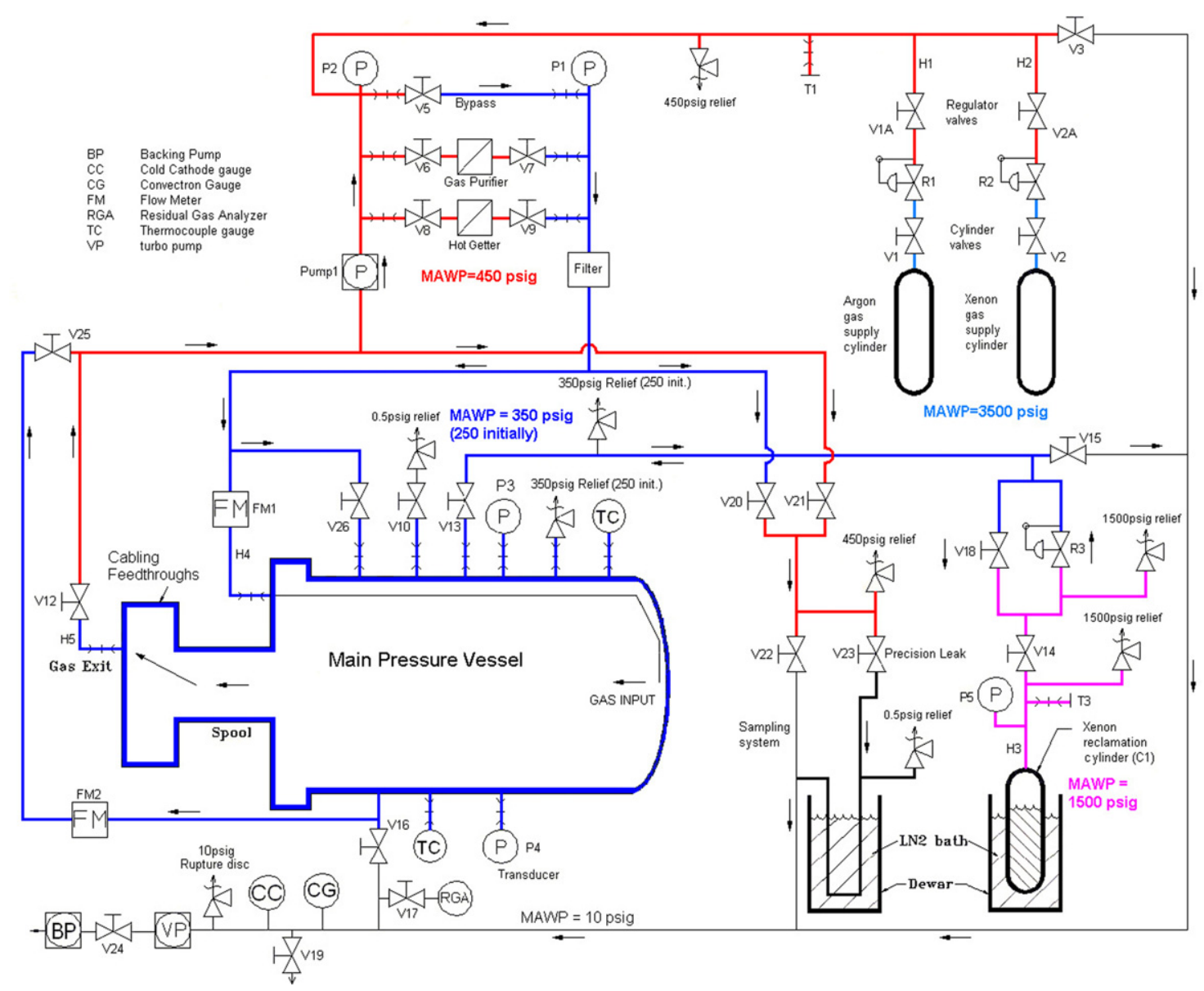

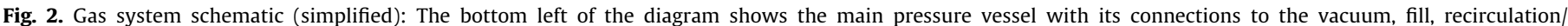
purification, sampling and reclamation components. 
and a residual gas analyzer (SRS model RGA100) and a room temperature secondary getter (SAES model MicroTorr MCP190).

A set of pressure relief valves (with different settings for the various parts of the pressure rated system) and a burst disk in the vacuum system protect the equipment and personnel from overpressure hazards.

The typical gas cycle during normal operation of the TPC consists of a vacuum step to $10^{-5}$ torr, followed by an argon purge and recirculation at $1 \mathrm{~atm}$, a second vacuum step to remove the argon and then the xenon fill from the reclamation cylinder. After the fill, the recirculation and purification is started, monitored with gas flow meters (models Sierra Smart-Trak and Omega FM1700) and controlled with a variac that powers the recirculation pump to set the recirculation flow.

\subsection{Pressure vessel and feedthrough ports}

The main pressure vessel is an 8.71 stainless steel cylindrical shell of $20 \mathrm{~cm}$ diameter and $33.5 \mathrm{~cm}$ length with an elliptical head on one end and a custom gasketed Conflat flange (main flange) on the other. Half inch VCR ports on the side of the vessel are used for gas fill, recirculation flow, pressure and temperature gauges and pressure relief valves. A $5.9 \mathrm{~cm}$ diameter (ID) port is used for vacuum pumping. On the main flange there are small ports with commercial high voltage feedthroughs (rated to $20 \mathrm{kV}$ and $17 \mathrm{~atm}$ ) with additional custom PTFE sleeves on the inside to increase the breakdown voltage when operating with high pressure xenon. A larger central port is used to connect to an octagonshaped vessel through a long tube with an internal source reentrance tube with a $2 \mathrm{~mm}$ thick endcap. Signal and highvoltage coaxial cables from the (in-vessel) PMT array pass through the axial extension tube and connect with 32-pin feedthroughs on the side ports of the octagonal vessel.

\section{3. $T P C$}

The field configuration in the TPC is established by five stainless steel meshes with $88 \%$ open area at a $z$ position of $0.5 \mathrm{~cm}$ (cathode buffer or PMT mesh), $5.5 \mathrm{~cm}$ (cathode or drift start mesh), $13.5 \mathrm{~cm}$ (field transition or EL-start mesh), $14.0 \mathrm{~cm}$ (anode or EL-end mesh) and $19.0 \mathrm{~cm}$ (anode buffer or ground mesh) from the PMT windows. Electroluminescence occurs between 13.5 and $14.0 \mathrm{~cm}$. The meshes are supported and kept tense by stainless steel frames made out of two parts and tensioning screws on the perimeter. The TPC side walls, made out of 18 individual rectangular assemblies $7.1 \mathrm{~cm}$ wide (and 5 and $8 \mathrm{~cm}$ length) connecting adjacent meshes (except around the $0.5 \mathrm{~cm}$ EL gap), serve the dual purpose of light cage and field cage (see Fig. 3). Each side wall assembly is made of a $0.6 \mathrm{~cm}$ thick PTFE panel and a ceramic support panel. The PTFE panels are bare on the side facing the active volume and have copper stripes parallel to the mesh planes every $0.6 \mathrm{~cm}$ on the other side. The bare PTFE serves as reflector for the VUV light (a 40\%-50\% Lambertian reflectivity was measured in Ref. [33]) to increase the light collection efficiency. Adjacent copper stripes are linked with $100 \mathrm{M} \Omega$ resistors to grade the potential and produce a uniform electric field. The ceramic support panels are connected, mechanically and electrically, to the outer perimeter of the mesh support frames and to the first and last copper stripes on their corresponding PTFE panel. High voltage connections to establish the TPC fields (HHV) are made directly to the mesh frames.

Six short PEEK rods going through holes on the mesh frames' vertices secure the anode and anode buffer meshes to the main vessel flange. Three PEEK c-clamps (on alternate sides of the hexagon) with grooves to prevent HV surface breakdown attach, in turn, the anode mesh to the field transition mesh maintaining a gas gap of $0.5 \mathrm{~cm}$. Finally, six PEEK rods support the field transition, cathode and cathode buffer meshes as well as the 19-PMT array. Mechanical tolerances obtained are better than $1 \mathrm{~mm}$ throughout the TPC geometry with better tolerances on the electroluminescent gap between 13.5 and $14.0 \mathrm{~cm}$.

In the initial implementation of the NEXT-DBDM TPC longer PEEK rods supported the entire assembly going through holes in the anode and field transition mesh frames with PEEK spacers to maintain the EL gap. High voltage breakdown in the form of sparks across the gap during HV conditioning and at random times thereafter produced conductive paths on the insulator surface, requiring time consuming repair. The c-clamp solution

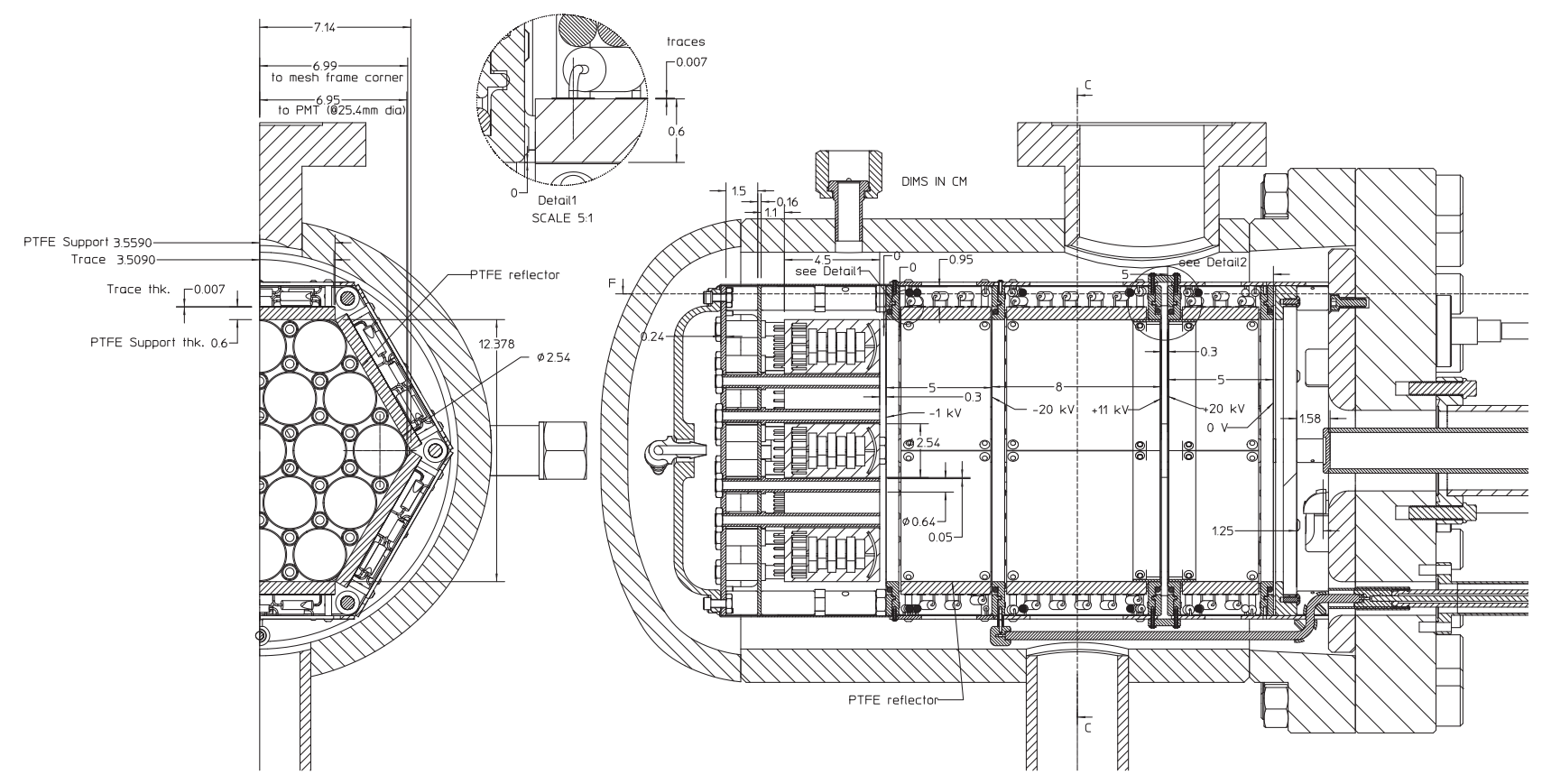

Fig. 3. Cutaway schematic of the TPC. 
described above made the TPC completely resilient to the unavoidable occasional sparking.

Clean gas from the purification system flows through an internal tube to an enclosed volume behind the PMTs and reaches the active volume through small dedicated PEEK tubes between the PMTs to exit the TPC through a port on the octagonal vessel at the end of the extension tube.

A wide range of HHV voltages were used to investigate the TPC performance dependence on drift electric field and on the $E / P$ in the EL region: cathode voltages from -4 to $-13 \mathrm{kV}$, field transition voltages from -1 to $-10 \mathrm{kV}$ and anode voltages from 1 to $13 \mathrm{kV}$.

\subsection{PMTs, readout and data processing}

The PMT array is composed of nineteen $2.54 \mathrm{~cm}$ diameter Hamamatsu 7378A PMTs. These PMTs, with fused silica windows, have a quantum efficiency of $\sim 15 \%$ for $172 \mathrm{~nm}$ xenon light. The PMTs were individually pressure tested to $19 \mathrm{~atm}$ (absolute) and no mechanical or performance failures were observed. The HV, typically about $-1 \mathrm{kV}$, is individually set to get a $\sim 10^{6}$ gain. The bases for the PMT array are implemented in a single hexagonal PCB board with surface mount components and with pin sockets that provide both mechanical support for the PMTs as well as the necessary electrical connections. The base has a total resistance close to $1 \mathrm{M} \Omega$, thus power dissipation is about $1 \mathrm{~W}$ per PMT. $1 \mu \mathrm{F}$ capacitors connected to the last dynode stages keep the gain constant during long EL light pulses. $200 \mathrm{pF}$ capacitors connected from the PMT anodes to ground stretch the pulse waveform such that all photoelectrons are properly sampled in the $100 \mathrm{MHz}$ digitizers.

PMT anode signals travel through $\sim 1 \mathrm{~m}$ long PTFE coaxial cables to the 32-pin feedthroughs and then to 8-channel Phillips777 amplifiers set to a gain of 40 . The amplified signals are then stretched and attenuated in a passive RCR circuit to reduce high frequency noise and to match the input range of the SIS3302 16-bit digitizers that sample the individual PMT signals at 100 mega-samples per second. PMT waveform data, typically 16,384 samples or $163.84 \mu \mathrm{s}$, are readout through an SIS3150 USB to VME interface to a Linux server for processing, analysis and storage. The overall system noise is such that individual photoelectrons can be detected and the instantaneous (10 ns) dynamic range per PMT is 1-to-200 photoelectrons. Custom-written DAQ software is used to control the data acquisition.

The trigger is designed to identify S2 EL pulses which have much larger area than the S1 pulses. The signal from a single PMT is integrated by a $12 \mu$ s peaking-time shaping amplifier and then sent to a discriminator. This scheme effectively provides an S2 energy threshold. The trigger is used as a stop signal (with a stop delay) for the digitizers such that there are, typically, $80 \mu \mathrm{s}$ of waveform data preceding the first S2 pulse and $80 \mu$ s after. Since the maximum drift time is about $80 \mu$ s, this permits the search of the $\mathrm{S} 1$ pulse in the offline analysis while ensuring that all the S2 light from one event is measured.

After a block of 512 events is collected with about 100 MBytes in raw DAQ data format, an automated process unpacks the data, applies calibration constants to the individual PMTs and executes the analysis code based on ROOT [34] and FMWK [35]. The analysis code finds S1 and S2 pulses, computes energies, times and positions, discards the individual PMT waveforms, and outputs the results in 1 MBytes ROOT data trees. The voluminous raw data is archived in long term storage.

\subsection{Controls and monitoring}

All system controls (except the single PMT HV power supply), such as HHV voltage settings and current limits and recirculation flow, are done manually. The three HHV voltages and currents, the PMT HV power supply voltage, the pressure inside the TPC vessel, the temperature at two points inside the TPC and the room temperature are automatically and continuously recorded for monitoring and to aid in offline data analysis. The recirculation flow is recorded manually.

\section{Data analysis}

In the first step of the data analysis the individual PMT waveforms are changed to photoelectron units using calibration constants from dedicated low occupancy single photoelectron runs with short LED light pulses. A sum waveform is then computed from a sample-by-sample addition of the 19 PMTs waveforms and the baseline of the sum waveform is obtained. A search for S1 and S2 pulses that cross a threshold follows. This threshold is determined from the value of the baseline noise. Pulses are defined as S1 candidates if they are less than 500 ns wide and have a maximum rise time (defined as the time from the start of the pulse to the location of the peak amplitude) of $100 \mathrm{~ns}$. All other pulses are considered S2 candidates. Individual pulses are integrated and the largest $\mathrm{S} 1$ candidate is assumed to be the event's start time and the others are discarded. All S2 pulses that follow the chosen S1 are considered part of the event while the ones preceding it are discarded. An event is considered valid if it has an S1 pulse with at least one associated S2 pulse. Figs. 4 and 5 show typical valid events from $662 \mathrm{keV}{ }^{137} \mathrm{Cs}$ gamma ray interactions.

During the automatic analysis of the data, the electron attachment lifetime of the gas is not yet known. In order to enable the offline correction of these charge losses without the data-heavy PMT waveforms the ten first moments of the S2 charge distribution $\int q(t) t^{n} d t$ with $n=0,1, \ldots, 9$ are calculated where $t$ is the time interval since the $\mathrm{S} 1$ start time pulse.

An average $x-y$ position for the event is calculated from an S2-charge weighted average of the PMT positions. The event is also time split into equal-charge slices. For each slice an average

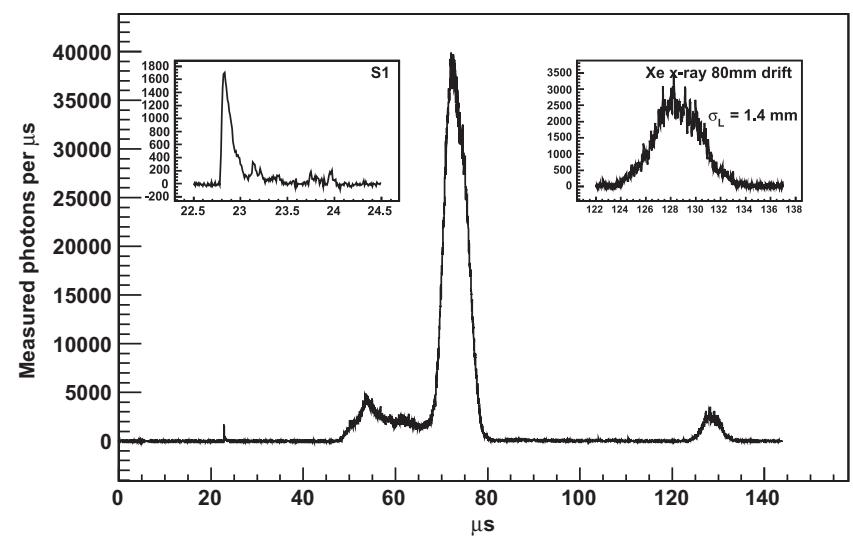

Fig. 4. Typical full energy $662 \mathrm{keV}$ gamma ray event waveform: the sum of the previously calibrated waveforms of the 19 photomultipliers is shown. The S1 pulse (shown in detail in the left inset) due to xenon scintillation is short and with $\mathrm{O}(200)$ measured photons. Two S2 pulses caused by electroluminescence of xenon from ionization electrons follow. The first with $\sim 270,000$ measured photons is likely due to a $630 \mathrm{keV}$ electron from the photoelectric interaction of the $662 \mathrm{keV}$ gamma ray; the pulse structure reflects the ionization density of the track along its $\sim 2.5 \mathrm{~cm}$ long projection on the $\operatorname{drift}(z)$ direction. The second (shown in detail in the right inset) with $\sim 12,000$ measured photons is likely due to the interaction, a few $\mathrm{cm}$ away, of a $30 \mathrm{keV}$ xenon X-ray following the photoelectric process; since the $30 \mathrm{keV}$ energy deposition is nearly point-like the pulse shape is gaussian with a $\sigma_{L}$ of $1.4 \mathrm{~mm}$ set by the longitudinal diffusion of the electrons during the $\sim 8 \mathrm{~cm}$ drift. This event is from a data run taken at $10 \mathrm{~atm}$ with a $0.16 \mathrm{kV} / \mathrm{cm}$ drift electric field and an $E / P$ of $2.1 \mathrm{kV} /(\mathrm{cm}$ atm) in the EL region. 


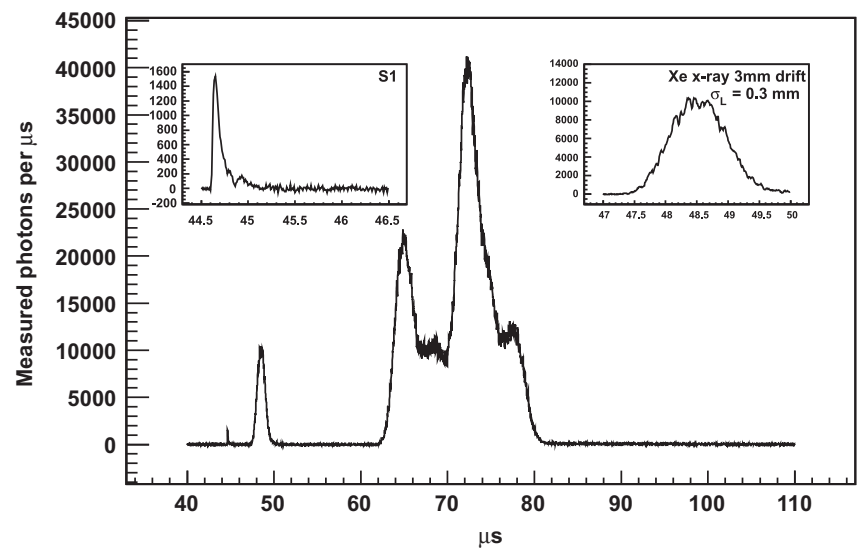

Fig. 5. A second typical full energy event: In this event from the same data run as in Fig. 4 the xenon X-ray interacted close to the EL region. The ionization electrons from it drifted for just $3 \mathrm{~mm}$ and underwent a correspondingly small longitudinal diffusion $\sigma_{L}$ of $0.3 \mathrm{~mm}$.

$x-y$ position is computed from the PMT light distribution. This provides $x-y-z$ positions for well separated energy depositions such as from Compton scatters or from X-rays following photoelectric interactions.

\section{Results}

\subsection{PMT performance}

As shown in formulas (2) and (3) the energy resolution achievable in an electroluminescent TPC depends on the precision with which individual photons can be measured. System noise and the variance from the avalanche multiplication in the PMT (mostly from the first dynode stages) contribute to the spread of the charge measured for photoelectrons. We found, however, that the charge fluctuations due to PMT after-pulsing are the dominant factor in the photoelectron charge resolution. After-pulsing caused by the occasional ionization of residual gas molecules in the PMT vacuum volume can produce delayed pulses with large charges (10-20 photoelectron charges are not uncommon). Dedicated LED data runs with light pulses less than 100 ns long (first afterpulse peaks from hydrogen and helium ions appear at 200 and 300 ns respectively for these small PMTs) were thus taken to assess the charge-variance for each PMT. The typical value was found to be $\sigma(Q) / Q=1.2$ thus $\sigma_{\mathrm{pd}}^{2}=1.44$, with small PMT-to-PMT variations.

\subsection{Position measurement}

The NEXT-DBDM TPC configuration with the PMT array $13.5 \mathrm{~cm}$ from the EL region does not permit detailed track reconstruction in the $x-y$ plane. Still, the position reconstruction achievable allows the fiducialization of pulses to select events/ pulses within regions of the TPC with uniform light collection efficiencies. The position reconstruction for isolated $50-100 \mathrm{keV}$ energy depositions shown in Fig. 6 displays the hexagonal boundary of the TPC. A scaling factor of $\sim 30$ and an $x-y$ offset are needed to convert charge-weighted average $x$ and $y$ positions to true TPC $(x, y)$ coordinates. The non-uniformity observed in Fig. 6 (with more density of points near the vertices of the hexagonal light cage and in the center) is due to the inaccuracies of the simple charge-weighting algorithm. A detailed Monte Carlo simulation of the experimental setup, including the EL light propagation and the optical properties of the detector

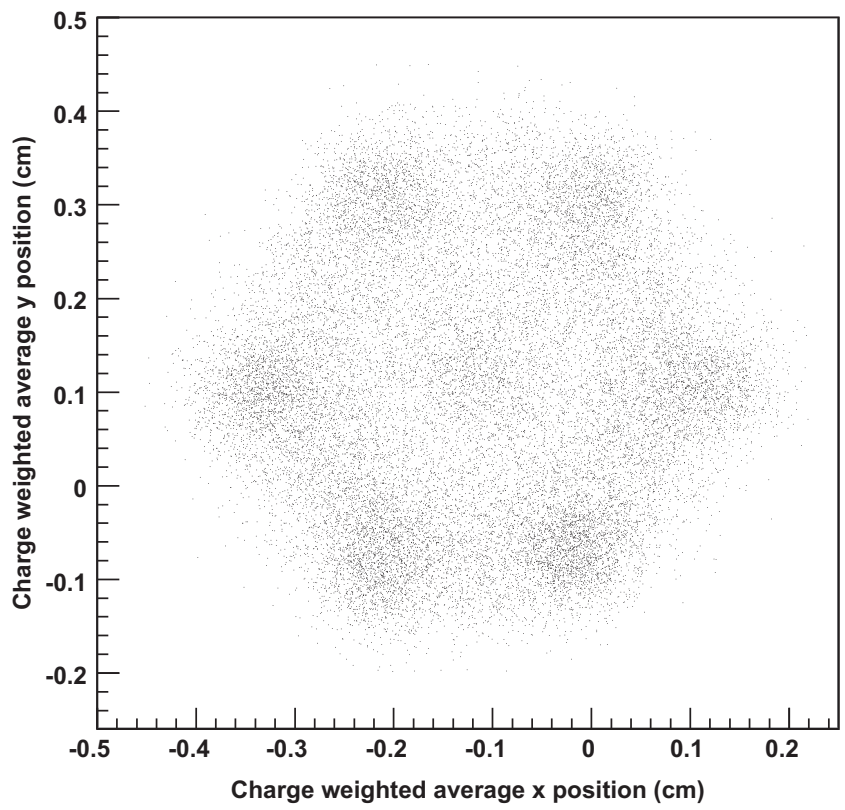

Fig. 6. Position reconstruction: The charge-weighted average of the 19 PMT positions is used for $x-y$ reconstruction. Events with energy depositions between 50 and $100 \mathrm{keV}$ were selected; at these energies ionization tracks extend for just a few $\mathrm{mm}$. The edges of the hexagonal area correspond to the TPC cross-section. Due to the spatial uniformity of the PMT plane illumination a scaling of factor of $\sim 30$ (not applied here) is needed to obtain true $x-y$ positions from these chargeweighted averages. These data were taken with the ${ }^{137} \mathrm{Cs}$ source, at $10 \mathrm{~atm}$ and with $E / P$ of $2 \mathrm{kV} /(\mathrm{cm}$ atm) in the EL region.

components shows the same non-uniformities, a nearly linear correspondence between true and reconstructed positions and the same $\sim 30$ scaling factor between them. The $x-y$ offset, on the other hand, is unique to the TPC data and is due to small PMT-toPMT differences in quantum efficiency and gain (not fully removed by the dedicated calibration).

Several data runs were taken with LED light pulses of various intensities to determine the position resolution for point depositions of charge as a function of the amount of light produced and detected. The resolution is derived from data with light pulses from a $378 \mathrm{~nm}$ LED located behind the PTFE backplane, which diffuses its light, and illuminates the PMT plane from a distance of about $19 \mathrm{~cm}$. For each LED intensity a fit of the radial distribution of the reconstruction position is performed. The fit function is the radial part of a two-dimensional Gaussian. Fig. 7 shows the obtained position resolutions which approximately follow the expected $1 / \sqrt{N}$ dependency on the photon statistics. Point-like depositions of charge, such as those from xenon X-rays, can thus be reconstructed in $x-y$ with better than $1 \mathrm{~cm}$ resolution if at least 10,000 EL photons are detected from them.

\subsection{Energy measurement}

The energy measurement is derived from the summed waveform (in photons detected) of all S2 pulses following the event S1 pulse. Fig. 8 shows a calibrated spectrum obtained from the ${ }^{137} \mathrm{Cs}$ source collimated with the gamma rays entering the chamber along its central axis. The full energy peak events in this data run have $\sim 270,000$ detected photons which, for $W_{\mathrm{i}}=24.8 \mathrm{eV}$, corresponds to about 10.1 photons detected per ionization (secondary) electron. Using the nominal EL gain $\eta=846$ from Eq. (5) the total number of photons produced is $846 \times 662,000 / 24.8 \simeq$ 22.6 million. From this, an S2-photon detection efficiency of $1.2 \%$ is deduced. 


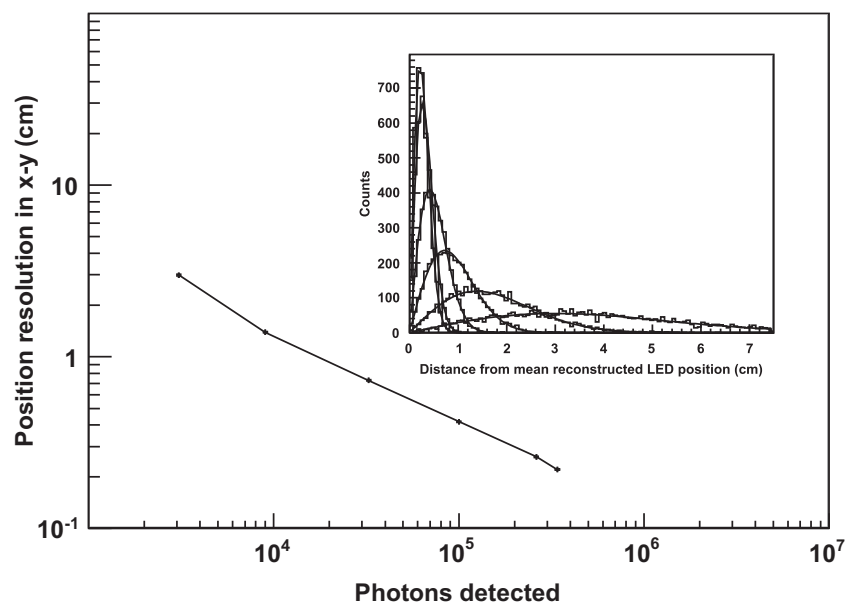

Fig. 7. Position resolution for LED light pulses: The position resolution in the $x-y$ plane as a function of the number of detected photons is shown. The inset shows the radial distributions (and fits) from which the individual resolution values were obtained.

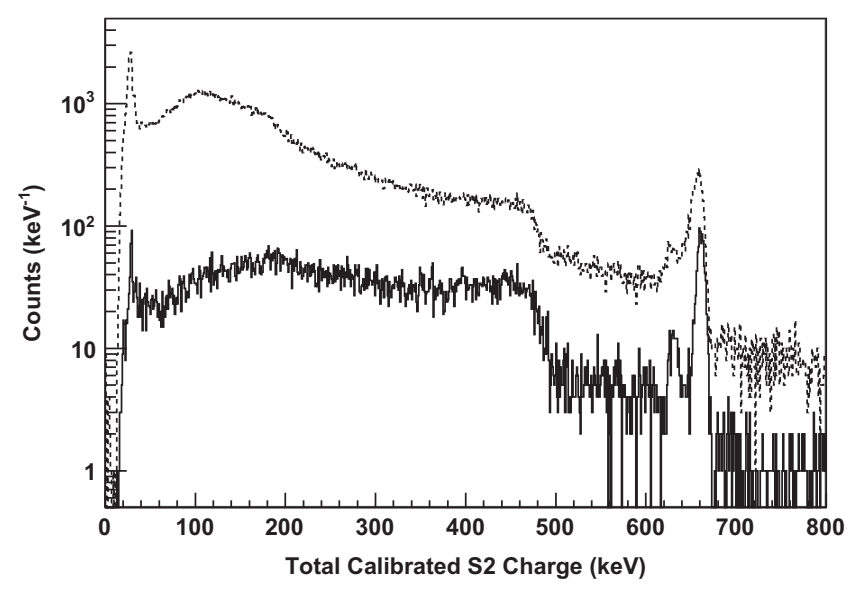

Fig. 8. Energy spectrum for ${ }^{137} \mathrm{Cs} 662 \mathrm{keV}$ gamma rays: The dashed line shows the calibrated spectrum (using the full energy $662 \mathrm{keV}$ peak alone) from a data run taken at 10 atm with a $0.3 \mathrm{kV} / \mathrm{cm}$ drift electric field and an $E / P$ of $2 \mathrm{kV} /(\mathrm{cm}$ atm) in the EL region. The $1 \mathrm{mCi}$ source is strongly collimated. Gamma rays enter the TPC along the drift axis $(z)$. Below the full energy peak at $662 \mathrm{keV}$, the smaller X-ray escape peak at $\sim 630 \mathrm{keV}$, the Compton edge at $477 \mathrm{keV}$ and the xenon X-ray peak at $\sim 30 \mathrm{keV}$ can be seen. The solid line is the same spectrum with a requirement that the reconstructed average position of an event be less than $\sim 1.2 \mathrm{~cm}$ from the TPC axis. The small feature near $184 \mathrm{keV}$ is due to Compton backscatters. The nosource background spectrum (not shown) has a broad peak near $100 \mathrm{keV}$.

While the spectrum was calibrated only using the $662 \mathrm{keV}$ line, the xenon X-rays peak appears at the correct energy (the two strongest X-ray lines are at $29.782 \mathrm{keV}$ and $29.461 \mathrm{keV} \mathrm{[28])}$ confirming the expected linearity of the energy measurement in the interval of interest here.

Attachment losses of drifting secondary electrons can be assessed from the full energy peak position as a function of the drift time of the events. Fig. 9 shows typical data for 10 and $15 \mathrm{~atm}$ with excellent electron lifetimes of 36 and $9 \mathrm{~ms}$, respectively. Less than $1 \%$ of the electrons are lost to attachment for the maximum $8 \mathrm{~cm}$ drift length. Assuming that the main source of electron attachment is due to residual $\mathrm{O}_{2}$, the measured electron lifetime at $10 \mathrm{~atm}$ corresponds to a residual oxygen component in the 2-4 part per billion range [36]. The lower lifetime at 15 atm is due to the quadratic pressure dependence of the 3-body attachment process rate.

The S2 photon collection efficiency is expected to vary somewhat as a function of the $x-y$ position because of solid angle and

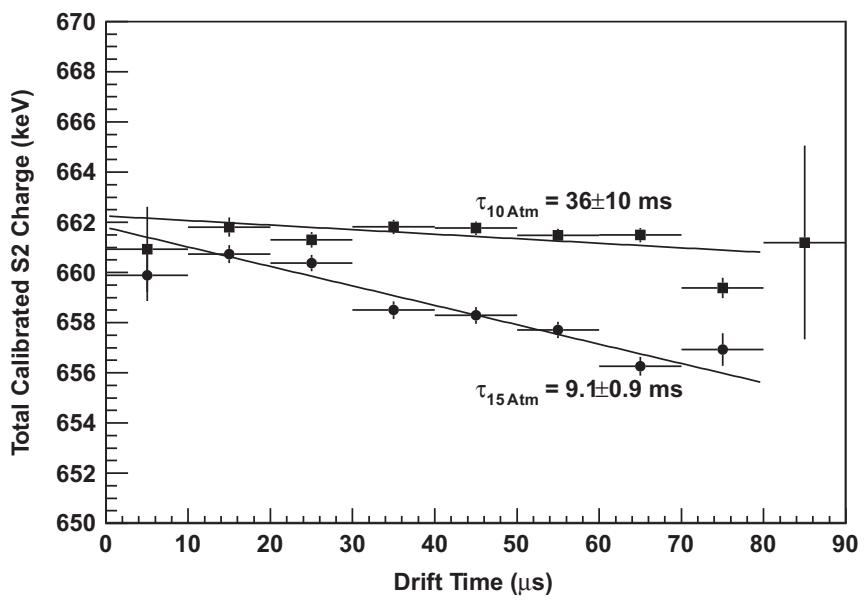

Fig. 9. Electron attachment losses: The average calibrated S2 charge versus the charge-weighted drift time is shown for full energy events. Attachment lifetimes are obtained from the exponential fits. The $10 \mathrm{~atm}$ data was taken with a $0.19 \mathrm{kV} / \mathrm{cm}$ field in the drift region and the $15 \mathrm{~atm}$ data with $0.59 \mathrm{kV} / \mathrm{cm}$.

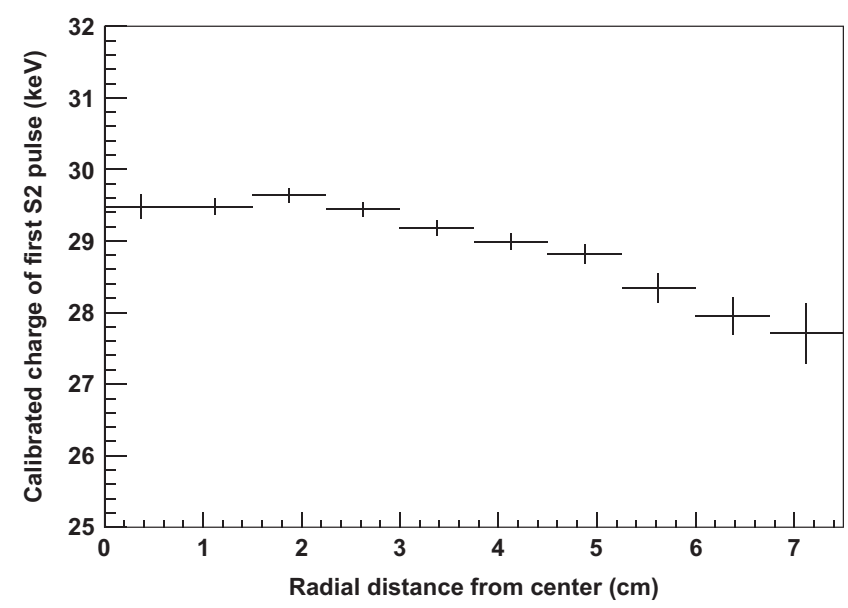

Fig. 10. Radial dependence of energy response: Xenon X-ray energy depositions were selected from full-energy $662 \mathrm{keV}$ events for which the first S2 pulse has energy in the $20-40 \mathrm{keV}$ range. The reduced response at larger radial positions (also observed in a detailed Monte Carlo simulation of the TPC) is due to geometric and optical effects of the EL light collection efficiency. These data were taken at $10 \mathrm{~atm}$ with a $1.0 \mathrm{kV} / \mathrm{cm}$ field in the drift region and $2.68 \mathrm{kV} /(\mathrm{cm}$ atm) in the $\mathrm{EL}$ region.

chamber optics (wall reflectivities, etc.). A sample of xenon X-ray energy depositions was used to measure the change in photon detection efficiency as a function of the radial position. Fig. 10 shows this dependence derived from a run in which individual $\mathrm{X}$-rays were reconstructed with $\sim 0.8 \mathrm{~cm}$ resolution in $x-y$. For the purpose of this measurement X-ray depositions can be considered point-like because $30 \mathrm{keV}$ electron tracks at these pressures extend typically for less than half a millimeter [37]. A nearly flat response is seen up to $2 \mathrm{~cm}$ radius followed by a linear decline in response towards the outer edge of the TPC where the collection is about $10 \%$ lower than in the center.

In the absence of detailed track imaging, the radial dependence of the detector response to point-like depositions affects the TPC energy resolution for extended tracks. Electron tracks from $662 \mathrm{keV}$ gamma interactions at the pressures of interest here have $2-4 \mathrm{~cm}$ spans and nearly random shapes due to the large coulomb multiple scattering in xenon. Fig. 11 shows the effect of a radial cut on the energy resolution of $662 \mathrm{keV}$ depositions and its efficiency. Since the collimated gamma rays enter the chamber along the main axis, events with a small average radial position 


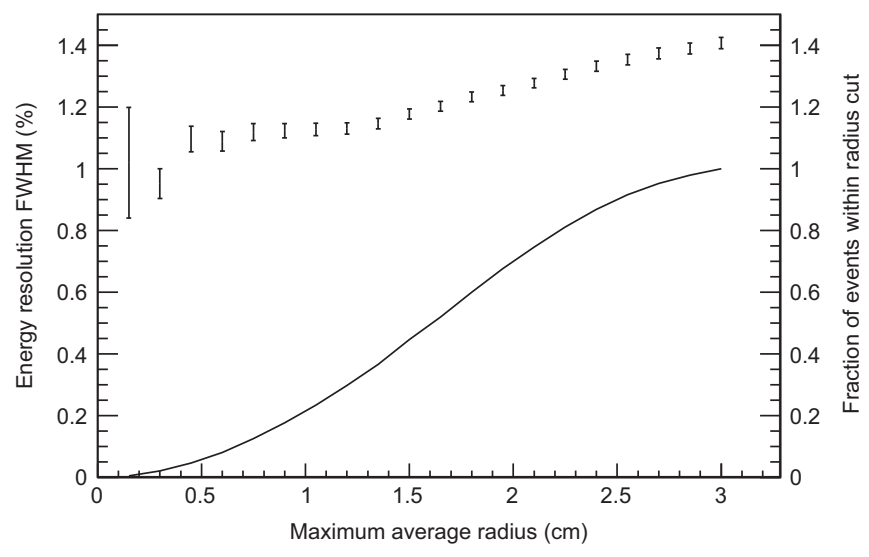

Fig. 11. Energy resolution dependence on radial cut: Full energy $662 \mathrm{keV}$ events are chosen and, for each, an average $x-y$ position is computed (and scaled to true TPC coordinates) using all of the S2 signal. The FWHM energy resolution (data points) is obtained from a gaussian fit to the peak after placing a cut on the radial position (the abscissa). The radial cut efficiency (solid line and right ordinate axis) is also shown. These data were taken at $10 \mathrm{~atm}$ with a $0.16 \mathrm{kV} / \mathrm{cm}$ field in the drift region and $2.1 \mathrm{kV} /(\mathrm{cm}$ atm $)$ in the EL region.

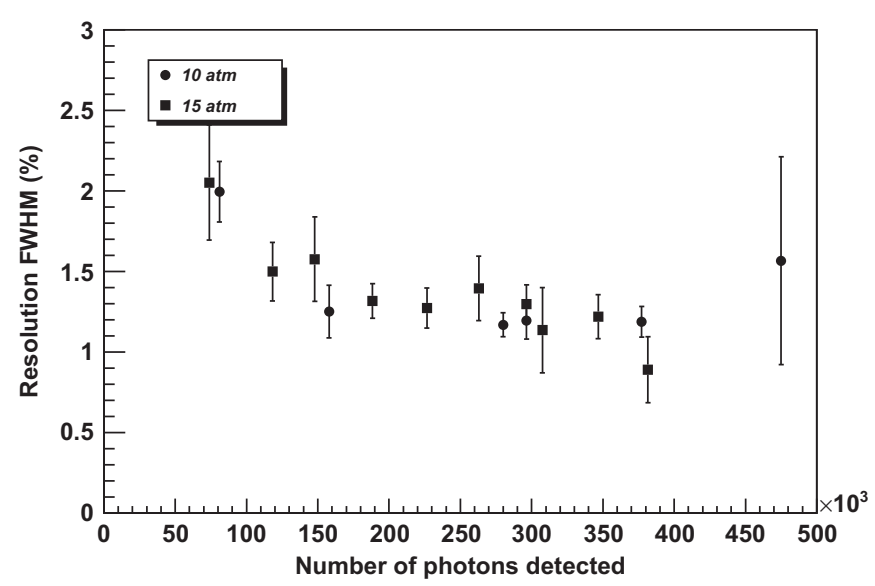

Fig. 12. Energy resolution dependence on amount of light detected: $662 \mathrm{keV}$ full energy peak resolutions are shown for data runs with different total S2 light yield (controlled primarily by the $E / P$ in the EL region). Energy spectra were obtained by selecting events reconstructed in the central $1 \mathrm{~cm}$ radius region and with an $\mathrm{S} 1$ signal with more than 100 measured photons and not more than 600 . A small electron drift-time-dependent attachment correction is applied. These data were taken with drift fields of $0.5-0.6 \mathrm{kV} / \mathrm{cm}$ at 10 atm and $0.16-0.72 \mathrm{kV} / \mathrm{cm}$ at $15 \mathrm{~atm}$.

have better energy resolution primarily because they span a smaller radial range.

Fig. 12 shows the improvement in the measured energy resolution for $662 \mathrm{keV}$ depositions with increased EL light yield. As seen in Eq. (2) and (3) the $G$ term dominates the resolution at low light yields ( $n_{\mathrm{pe}}$ small) while the intrinsic Fano term is the asymptotic resolution in the large light yield limit.

In Fig. 13 the energy spectrum in the $662 \mathrm{keV}$ full energy region obtained at $10 \mathrm{~atm}$ is shown. A $1.1 \%$ FWHM energy resolution was obtained for events reconstructed in the central $0.6 \mathrm{~cm}$ radius region. A small drift-time dependent correction for attachment losses with $\tau=13.9 \mathrm{~ms}$ was applied. The xenon X-ray escape peak is clearly visible $\sim 30 \mathrm{keV}$ below the main peak. For the spectrum taken at $15 \mathrm{~atm}$, shown in Fig. 14, a 1\% FWHM resolution was obtained. At this higher pressure the xenon X-ray is less likely to escape from the active volume and the escape peak almost disappears. This resolution extrapolates to $0.52 \%$ FWHM at $Q_{\beta \beta}=2.459 \mathrm{MeV}$ if the scaling follows a statistical $1 / \sqrt{E}$ dependence and no other systematic effect dominates.

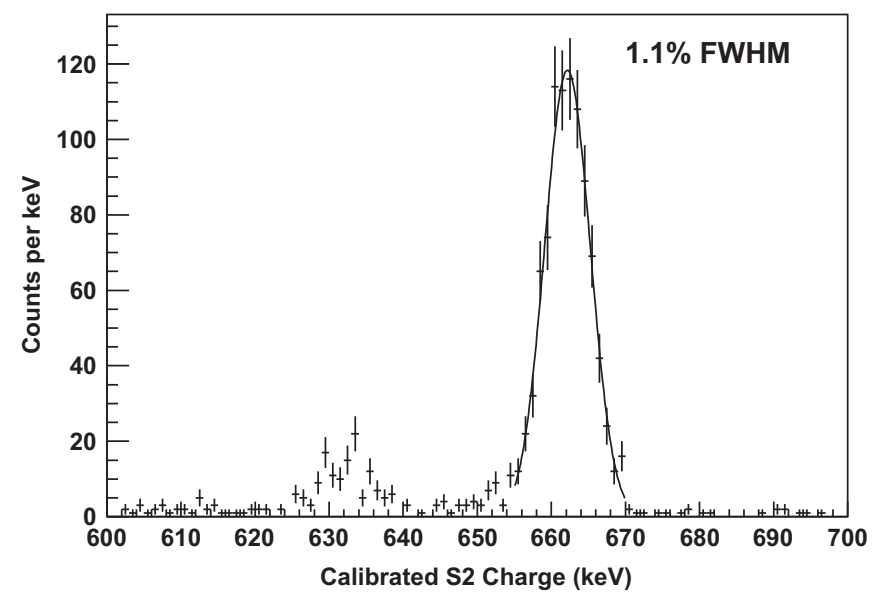

Fig. 13. Energy resolution at $10 \mathrm{~atm}$ for $662 \mathrm{keV}$ gamma rays: These data were taken at $10.1 \mathrm{~atm}$ with a $0.16 \mathrm{kV} / \mathrm{cm}$ field in the drift region and $2.08 \mathrm{kV} /(\mathrm{cm}$ atm) in the EL region. If assumed to follow a $1 / \sqrt{N}$ dependence this resolution extrapolates to $0.57 \%$ at $Q_{\beta \beta}=2.459 \mathrm{MeV}$.

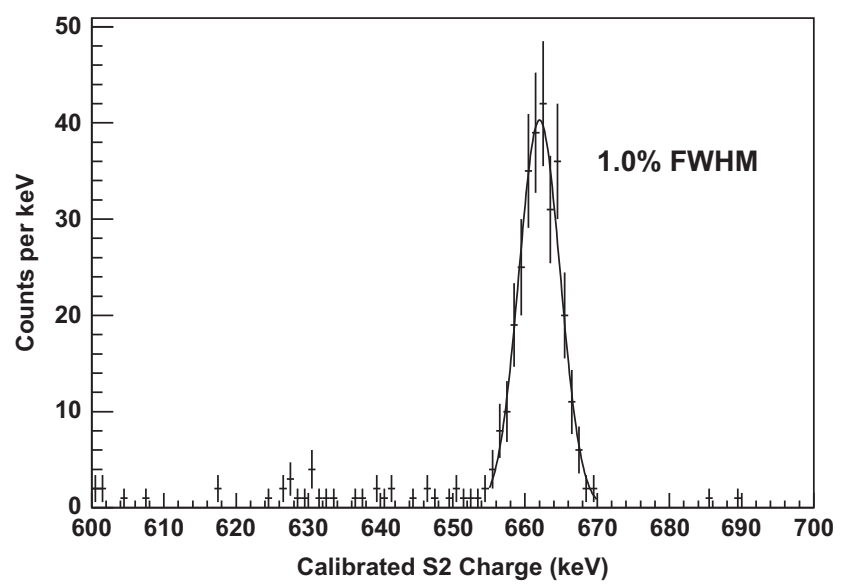

Fig. 14. Energy resolution at $15 \mathrm{~atm}$ for $662 \mathrm{keV}$ gamma rays: A $1.0 \%$ FWHM energy resolution was obtained for events reconstructed in the central $0.75 \mathrm{~cm}$ radius region. The attachment losses correction with $\tau=9.0 \mathrm{~ms}$ was applied. A PMT with a clear time varying response was removed from the measurement. These data were taken at $15.1 \mathrm{~atm}$ with a $0.59 \mathrm{kV} / \mathrm{cm}$ field in the drift region and $1.87 \mathrm{kV} /(\mathrm{cm}$ atm) in the EL region.

In order to study the EL TPC energy resolution at lower energies, full energy $662 \mathrm{keV}$ events that had a well separated $\mathrm{X}$-ray pulse reconstructed in the central $1.5 \mathrm{~cm}$ radius region were used. The energy calibration was done on the $662 \mathrm{keV}$ full energy peak and linearity with a zero intersect was assumed. Fig. 15 shows the energy spectrum obtained at 10 atm with a $5 \%$ FWHM resolution. The spectrum was fit to the sum of four Gaussians with relative positions and intensities fixed to the strongest xenon X-ray lines [28]. The absolute position of the anchor peak and the peaks' widths (all assumed the same) were obtained from the fit. The anchor peak is at $29.1 \mathrm{keV}$, less than $2 \%$ away from its nominal $29.6 \mathrm{keV}$ value.

Fig. 16 summarizes our measurements and understanding of the EL TPC energy resolution. The lower diagonal line represents the Poisson statistical limit from the measurement of a small fraction of the photons produced by the EL gain while the upper diagonal line includes the degradation (mostly from PMT afterpulsing) due to PMT response. The circle data points show the energy resolutions obtained for dedicated LED runs with varying light intensities per LED pulse. The LED points follow the expected resolution over the two decades range studied. The two horizontal 
lines represent the xenon gas nominal intrinsic resolution for 30 and $662 \mathrm{keV}$, respectively, and the two curved lines are the expected EL TPC resolutions with contributions from the intrinsic limit and the photons' measurement. Our $662 \mathrm{keV}$ data (squares) and xenon X-ray data (triangles) taken with various EL gains follow the expected functional form of the resolution but are $20 \%-30 \%$ larger possibly due to the $x-y$ response non-uniformity. Detailed track imaging from a dense photosensor array near the EL region, such as the one recently commissioned for the NEXT-DBDM prototype (see Section 8.1), will enable the application of $x-y$ position corrections to further improve the energy measurement.

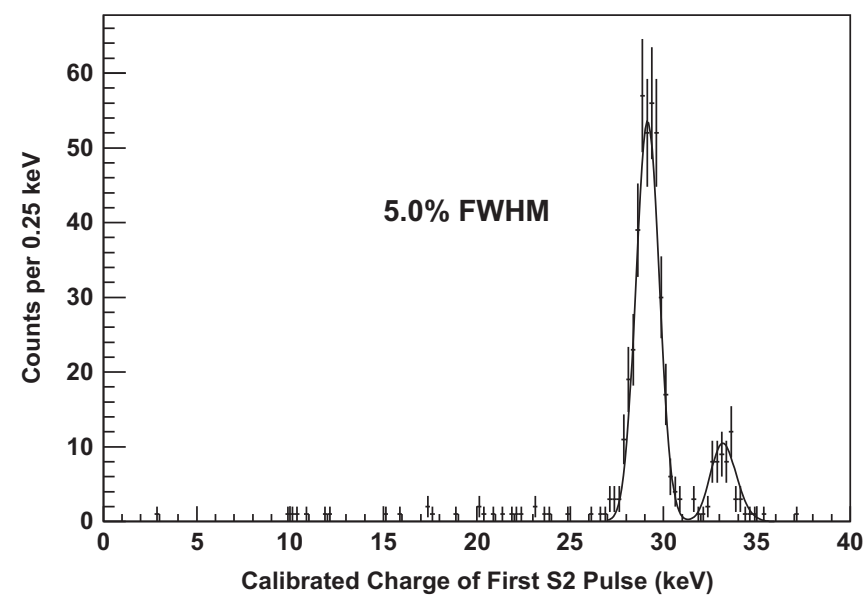

Fig. 15. Energy resolution at $10 \mathrm{~atm}$ for $30 \mathrm{keV}$ xenon X-rays: A 5\% FWHM energy resolution for $30 \mathrm{keV}$ was obtained. These data were taken at $10.1 \mathrm{~atm}$ with a $1.03 \mathrm{kV} / \mathrm{cm}$ field in the drift region and $2.68 \mathrm{kV} /(\mathrm{cm}$ atm $)$ in the EL region.
In Ref. [11], a study of the energy resolution for $662 \mathrm{keV}$ gamma rays at pressures near condensation (30 atm and above) using a xenon ionization chamber found an improvement of the resolution for increased drift fields. Asymptotic optimum resolutions were achieved only after applying $4 \mathrm{kV} /$ $\mathrm{cm}$ or larger fields. Since these large fields would be difficult to achieve in a detector with $1 \mathrm{~m}$ long drift region such as the one planned for NEXT-100, we investigated the resolution dependence on the drift electric field at $10 \mathrm{~atm}$. Fig. 17 shows no discernible dependence in the $0.16-1.03 \mathrm{kV} / \mathrm{cm}$ region investigated.

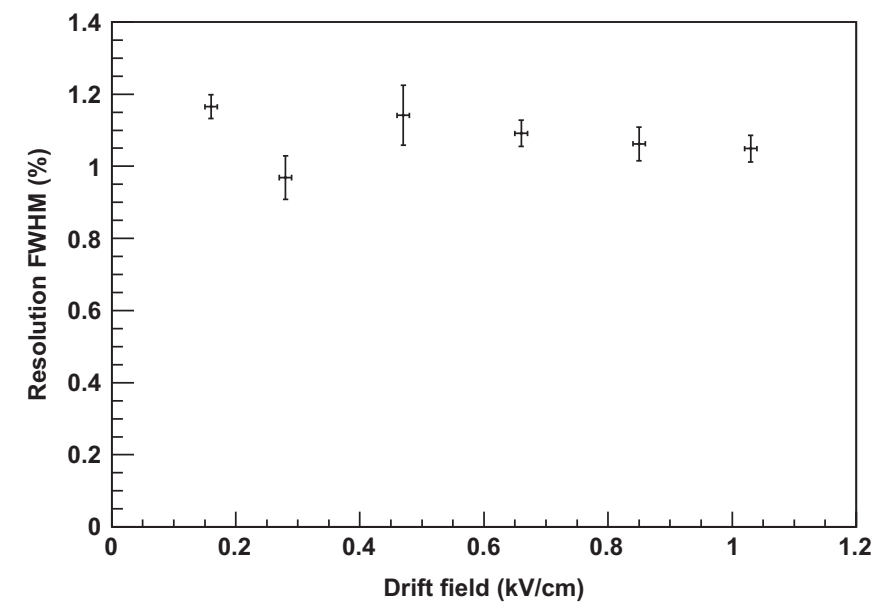

Fig. 17. Energy resolution dependence on drift field: These data were taken at $10.1 \mathrm{~atm}$ with $2.1-2.7 \mathrm{kV} /(\mathrm{cm}$ atm $)$ in the $\mathrm{EL}$ region. The energy resolution attained is largely independent of the drift electric field in the investigated range.

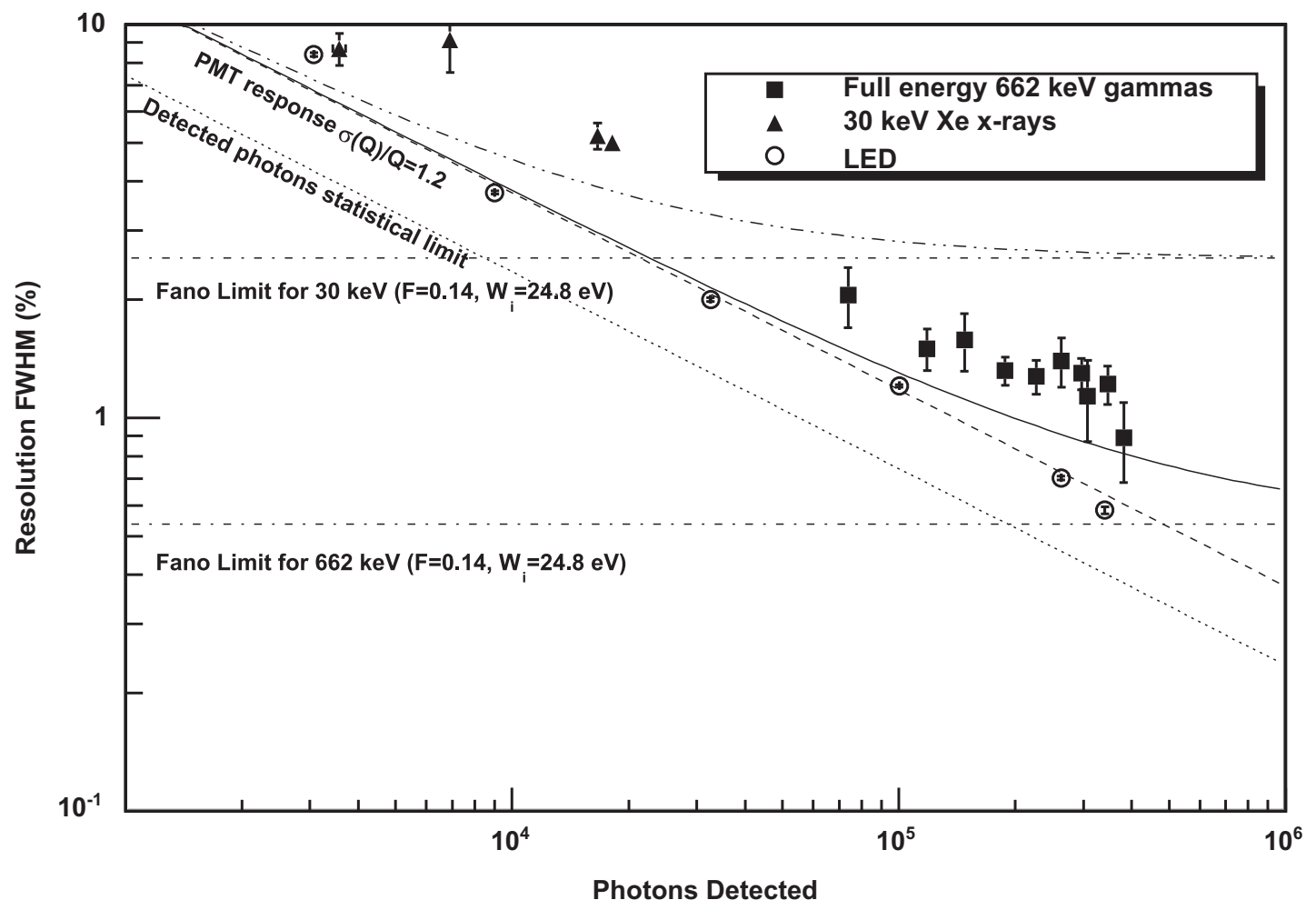

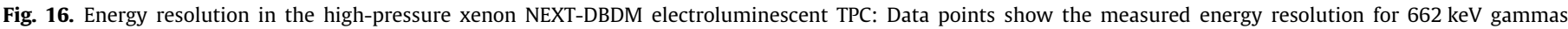

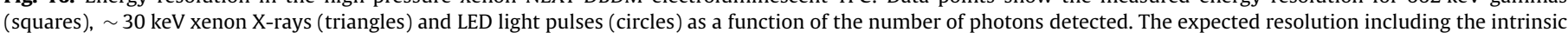

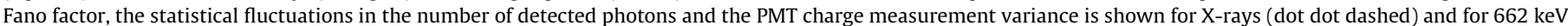
gammas (dot dot dot dashed). Resolutions for the $662 \mathrm{keV}$ peak were obtained from $15.1 \mathrm{~atm}$ data runs while X-ray resolutions we obtained from 10.1 atm runs. 


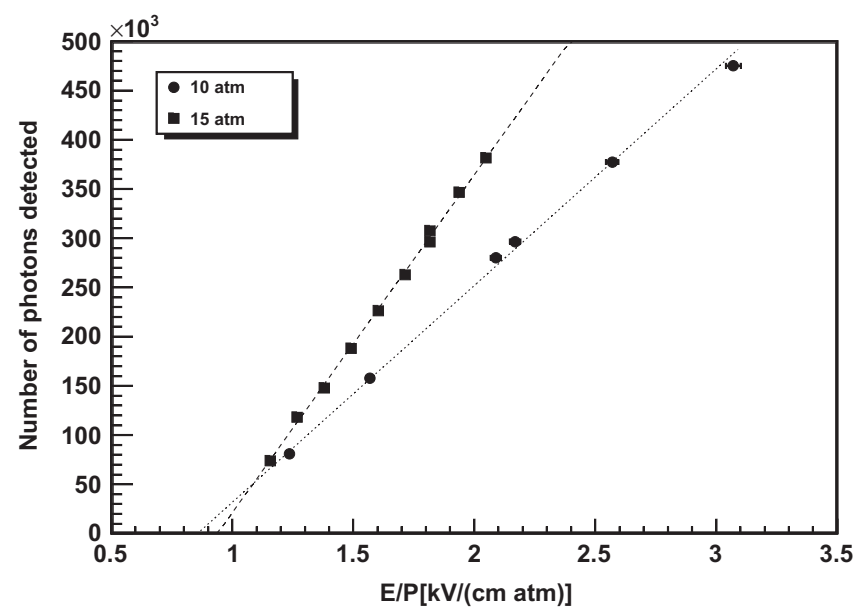

Fig. 18. Light yield dependence as a function of $E / P$ in the EL region.

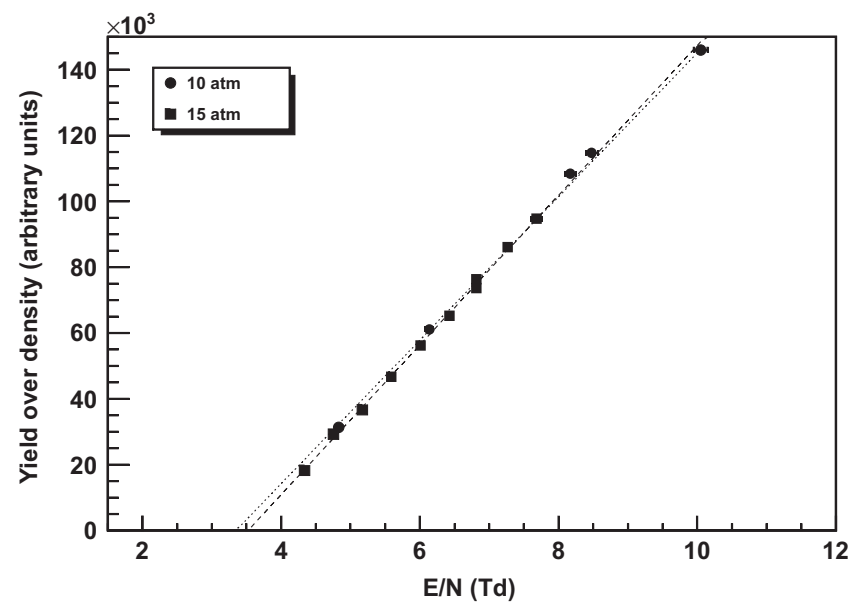

Fig. 19. Density normalized light yield vs. EL reduced field. The lines are the results of fits to a linear function for the $10 \mathrm{~atm}$ (dotted) and $15 \mathrm{~atm}$ (dashed) data points.

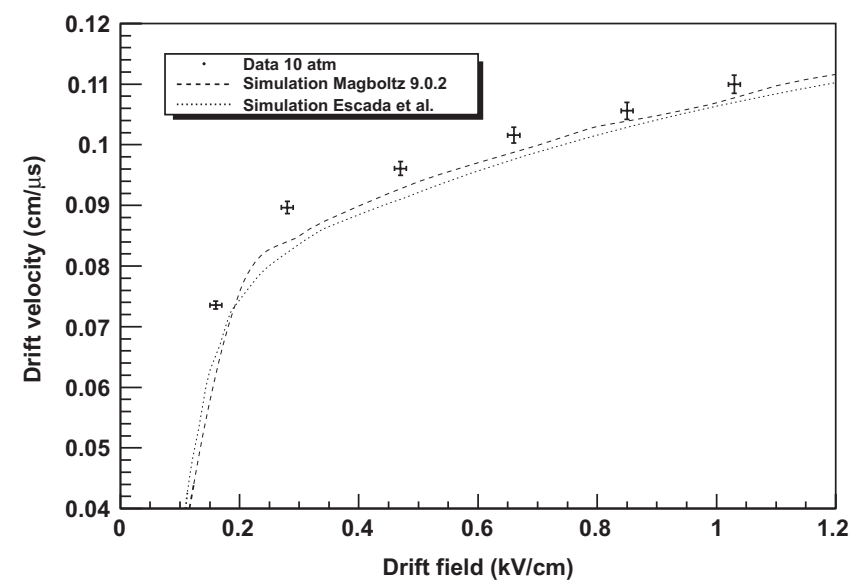

Fig. 20. Drift velocity dependence on drift field: The electron drift velocity obtained from the maximum drift times measured (which correspond to the full drift length of $8 \mathrm{~cm}$ ) for X-ray energy depositions. These data were taken at $10.1 \mathrm{~atm}$.

\subsection{EL TPC characterization: light yield, drift velocity and longitudinal diffusion}

While the study of the energy resolution achievable in the EL TPC was the main goal of this work, we pursued other

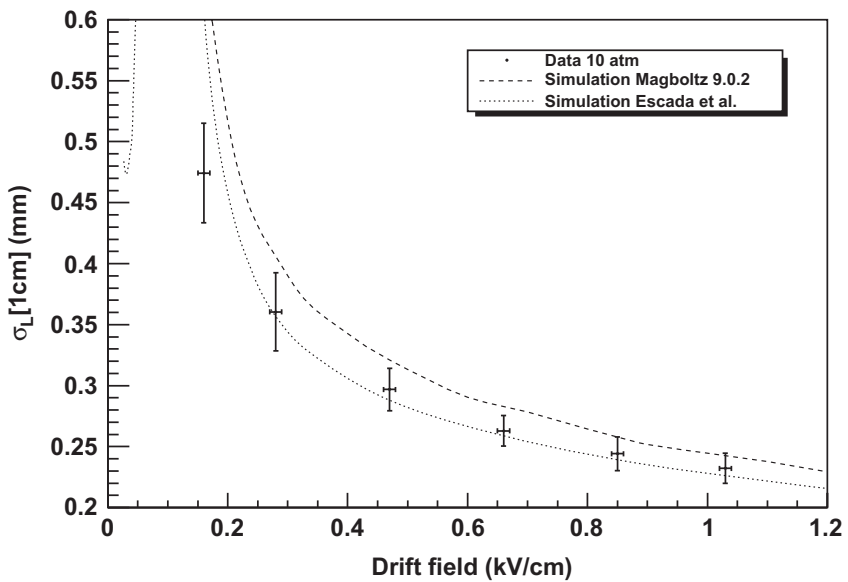

Fig. 21. Longitudinal diffusion dependence on drift field: The measured longitudinal diffusion is presented here as the $\sigma$ spread along the drift direction for a $1 \mathrm{~cm}$ drift. These data were taken at $10.1 \mathrm{~atm}$.

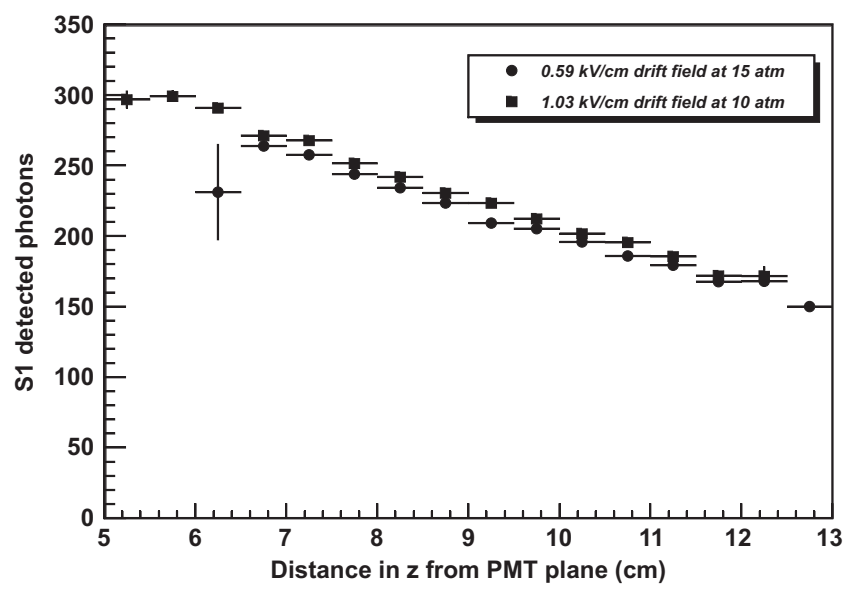

Fig. 22. S1 signal dependence on z position: For events with full energy $662 \mathrm{keV}$ depositions, the number of measured S1 photons is shown versus the average distance of the ionization track from the PMT plane (obtained from the S2 chargeweighted drift time and the measured drift velocity). Two runs are shown with $E / P$ in the drift region of 0.04 and $0.10 \mathrm{kV} /(\mathrm{cm}$ atm) with very similar S1 yield.

measurements as well as cross checks against previous results by others. Fig. 18 shows the linearity of the EL light yield as the $E /$ $P$ in the $0.5 \mathrm{~cm}$ EL region is varied from 1 to $2 \mathrm{kV} /(\mathrm{cm}$ atm $)$ at $15 \mathrm{~atm}$ and from 1 to $3 \mathrm{kV} /(\mathrm{cm}$ atm $)$ at $10 \mathrm{~atm}$. At these pressures xenon deviates from the ideal gas behavior at the $10 \%$ level. Therefore, the more appropriate variables to describe the process are density (N) and reduced electric field (E/N). Fig. 19 shows the density normalized EL yield as a function of the reduced field. As expected, points from the 10 and 15 atm data sets follow the same linear trend with consistent slope and threshold.

The electron drift velocity was obtained from the maximum drift times measured (which correspond to the full drift length of $8 \mathrm{~cm}$ ) for X-ray energy depositions. Fig. 20 shows the drift velocity measured for various drift fields along with two Monte Carlo calculations $[36,38]$ using up-to-date xenon-electron collision cross-sections. While the data points follow the trend in the calculations, $10 \%$ deviations are seen at the lower drift fields. Yet, the general agreement observed validates the pressure and field measurements as well as the drift field uniformity and the xenon purity.

The longitudinal diffusion was calculated from the time spread of EL light of X-ray depositions (see example fits in the upper right insets of Figs. 4 and 5) and using the measured drift velocity. 

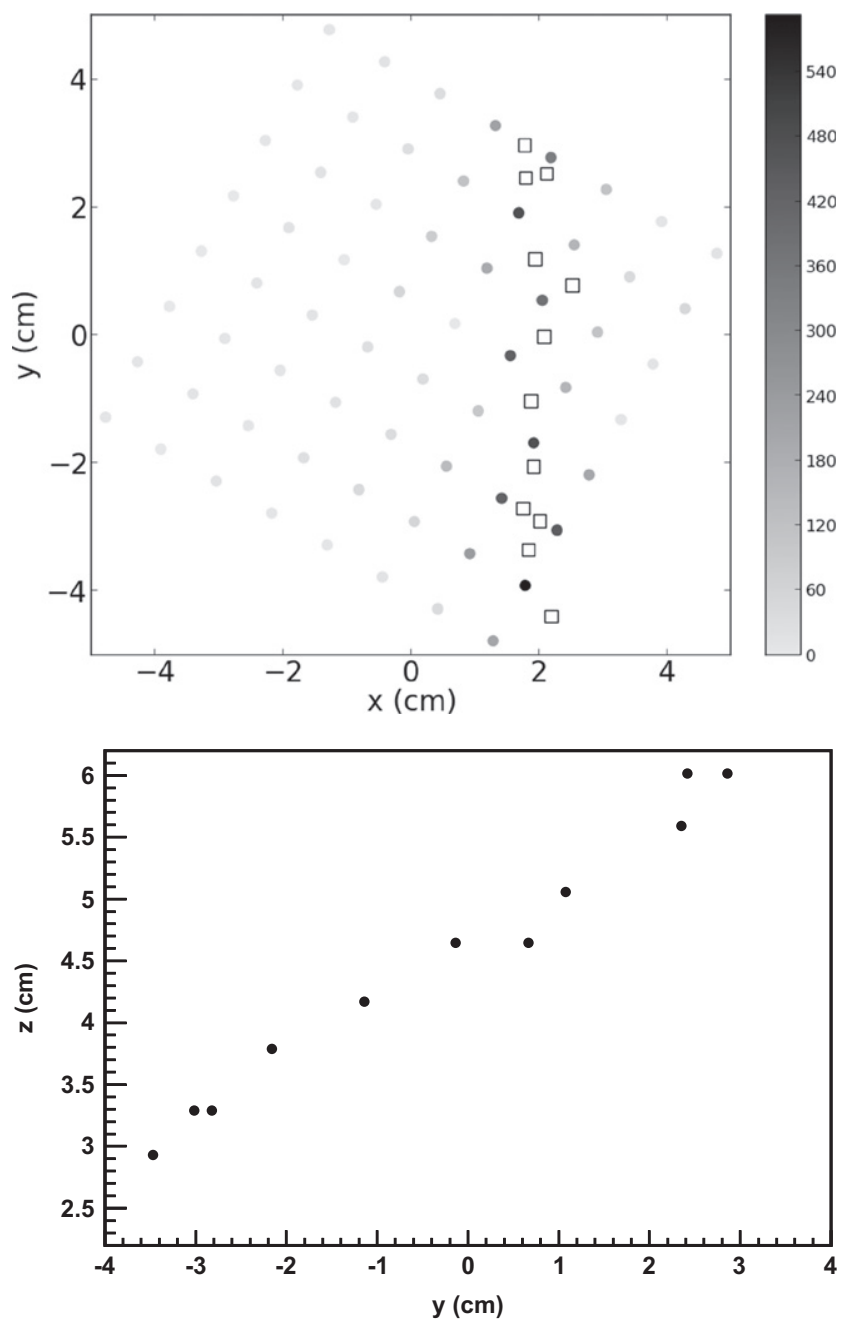

Fig. 23. Cosmic ray muon track measured in the $8 \times 8$ SiPM array near the EL region: Top: The 64 SiPMs covering an $8 \mathrm{~cm}$ by $8 \mathrm{~cm}$ area in the center of the TPC are shown as circles shaded relatively according to the total amount of light observed by each. The $x-y$ coordinates at each point in the reconstructed track are given by the squares. Bottom: The $z$ coordinate is calculated from the time delay between the SiPM signals and the event S1 with the known drift velocity.

For X-ray pulses with long drift times the longitudinal diffusion is the dominant source of pulse width with subdominant contributions from the transit time of electrons through the EL gap and from the ionization track length. Fig. 21 shows the dependence of the longitudinal diffusion on the drift field and the corresponding Monte Carlo calculations. Good agreement is observed between the measurement and the calculations with a preference for the lower diffusion model.

\subsection{S1 measurement}

Unlike the S2 light which is generated within a small $0.5 \mathrm{~cm}$ region in $z$, the $\mathrm{S} 1$ photons are produced throughout the entire TPC volume wherever the primary ionization happened. As a result, the S1 charge (number of photons detected) shows large event-by-event variations. S1 light collection efficiency is largest for events closer to the PMT array due to the larger solid angle. Fig. 22 shows the S1 signal's dependency on the average $z$ position of the event. Between 0.2 and $0.45 \mathrm{~S} 1$ photons are detected per keV of energy deposited. This S1 yield is consistent with the known $W_{s}$, the average energy loss required to liberate one primary scintillation photon in gaseous xenon [39-41], and the photon collection and detection efficiency in our TPC. S1
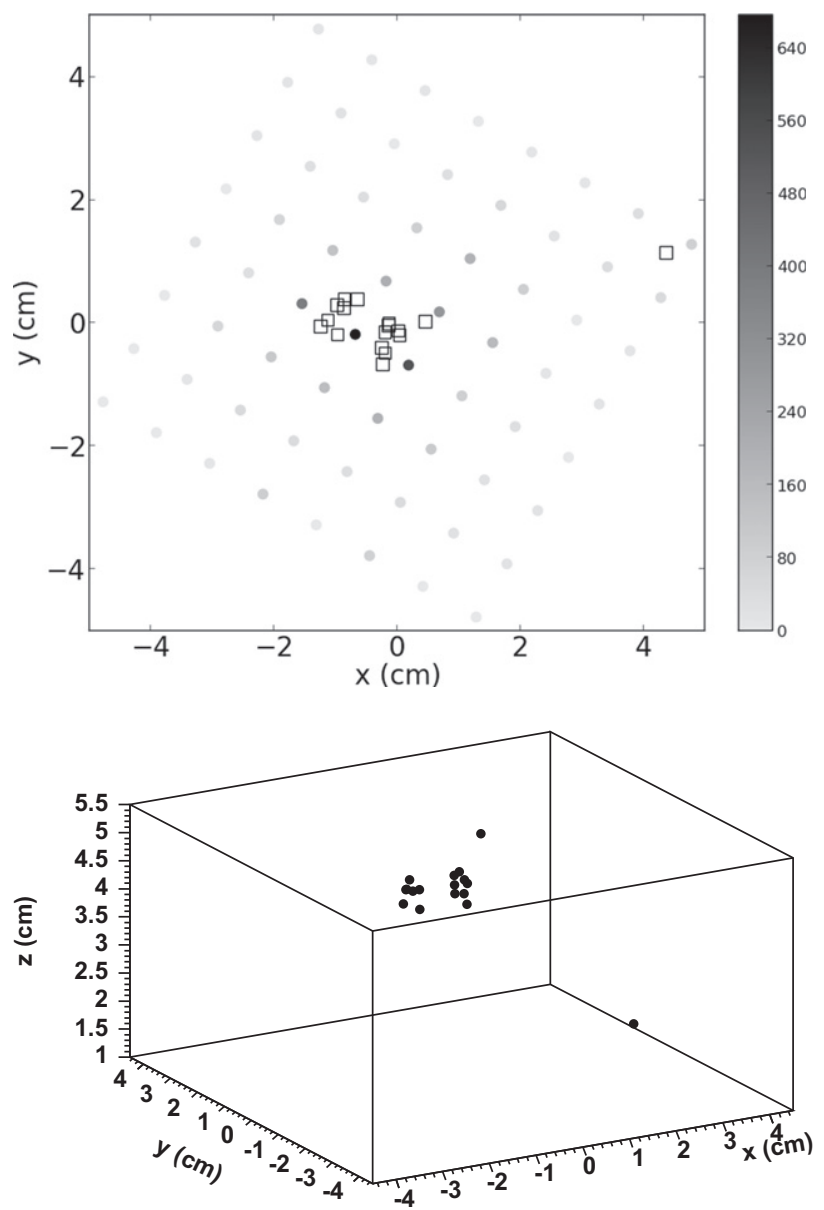

Fig. 24. Gamma ray event with photoelectron track and X-ray. Top: the SiPM hits and the reconstruction points in the central region are caused by the $630 \mathrm{keV}$ photoelectron track. The isolated reconstruction point near $(45,12)$ is due to the $30 \mathrm{keV}$ X-ray. Bottom: 3D reconstruction of the event.

photons are produced from direct excitations of xenon atoms by the ionizing particle and by ion-electron recombinations. The latter component is, in general, field and pressure dependent. Fig. 22 shows less than 5\% differences between the S1 yield of runs taken with reduced fields of 0.04 and $0.10 \mathrm{kV} /(\mathrm{cm}$ atm) in the drift region. We conclude that, as expected, any recombination effects are small for electron energy depositions of interest here in the range of pressures and fields investigated.

\section{Further developments and plans}

\subsection{Tracking plane}

Since the completion of the energy resolution studies reported above we have finished the construction and installation of the sensor array for the tracking plane. It is a $1-\mathrm{cm}-$ pitch 8 by 8 square pattern array of Hamamatsu S10362-11-025P silicon photomultipliers (SiPMs) each with $1 \mathrm{~mm}^{2}$ active area. The SiPMs are coated with a thin film of tetraphenyl butadiene (TPB) and placed in the center of the hexagonal plane $2 \mathrm{~mm}$ behind the electroluminescent region of the TPC. The SiPMs produce an electronic pulse for each detected photon, and the TPB serves to shift the incoming $172 \mathrm{~nm}$ light from xenon to visible light detectable by the SiPMs. Details of the SiPM performance and coating are given in Ref. [15]. The low power electronics to amplify, shape and multiplex the signals from the array was custom built to fit inside the pressure vessel. The 
digitization of the multiplexed signals is done outside the vessel with the same digitizers as for the PMT system.

Details of the operation, calibration and analysis of the SiPM array for the tracking plane will be reported in a future publication. Here, we present first results that demonstrate the system's ability to produce detailed track images. The top panel in Fig. 23 shows the relative amounts of light detected in each SiPM from the electroluminescence produced by an externally tagged cosmic ray muon that traversed the active volume of the TPC. A reconstruction based on a likelihood fit of the time sliced data shows the straight muon trajectory. The RMS error in position is estimated to be approximately $2.1 \mathrm{~mm}$ for individual reconstructed points. The bottom panel the figure shows the $z-y$ projection of the reconstructed points obtained using the time projection of the TPC. A clear 3D track is obtained even for this reconstruction that uses raw (not calibrated) SiPM signal waveforms.

Fig. 24 shows the $2 \mathrm{D}$ and $3 \mathrm{D}$ projections of the track reconstruction of a full energy $662 \mathrm{keV}$ gamma ray event. The $30 \mathrm{keV}$ $\mathrm{X}$-ray deposition is well separated in $z$ (time) and in $x-y$ about $5 \mathrm{~cm}$ away from the $3 \mathrm{~cm}$ long photoelectron track that shows the expected multiple scattering.

Track information from this SiPM system will be used to map and correct the $x-y$ energy response of the PMT plane with the goal of demonstrating a near-intrinsic energy resolution over a large part of the active volume of the detector. Reconstructed tracks will also be used to study the topological information that will enable, in the NEXT-100 detector, the identification of $0 v \beta \beta$ candidate events while rejecting single and multi-track background events due to gamma rays from residual radioactivity in detector and surrounding materials.

\subsection{Gas additives and nuclear recoil studies}

A research program is underway to study gas additives to optimize the detector performance. We are investigating the properties of high pressure xenon mixtures with $\mathrm{CF}_{4}, \mathrm{CH}_{4}$ and TMA (trimethylamine). These studies focus on diffusion (for track imaging), electroluminescence and primary scintillation yield, and energy resolution. Results of those studies will be reported elsewhere.

We are also investigating the high pressure EL TPC response to low energy nuclear recoils. $\mathrm{O}(10 \mathrm{keV})$ nuclear recoils are expected from WIMP (Weakly Interacting Massive Particle) dark matter elastic interactions with the xenon target. These studies, using $\mathrm{MeV}$ neutron sources, aim to characterize the S2/S1 discrimination between electron and nuclear recoils and to optimize the light collection from the feeble nuclear recoil S1 signals.

\section{Conclusions}

We presented the design, data and results from the NEXTDBDM high-pressure gaseous natural xenon electroluminescent TPC that was built at the Lawrence Berkeley National Laboratory. Energy resolutions of 1\% FWHM for $662 \mathrm{keV}$ gamma rays were obtained at 10 and $15 \mathrm{~atm}$ and 5\% FWHM for $30 \mathrm{keV}$ fluorescence xenon X-rays. The main contributions to the energy resolution were studied and are well understood and thus justify the $1 / \sqrt{E}$ extrapolation to a $0.5 \%$ FWHM resolution for the $2459 \mathrm{keV}$ hypothetical neutrino-less double beta decay peak. This energy resolution is a factor 7-20 better than that of the current leading $0 v \beta \beta$ experiments using liquid xenon. We presented also first results from a track imaging system consisting of 64 silicon photo-multipliers recently installed in NEXT-DBDM that, along with the excellent energy resolution, demonstrates the key functionalities required for the NEXT-100 $0 v \beta \beta$ search. The construction of the NEXT-100 detector with $90 \mathrm{~kg}$ of ${ }^{136} \mathrm{Xe}$ detector has began and first data is expected for 2015.

\section{Acknowledgments}

We thank Adam Bernstein and Mike Heffner for the loan of the high pressure and storage vessels from LLNL (under LLNL loan 101-3026). This work was supported by the Director, Office of Science, Office of Basic Energy Sciences, of the U.S. Department of Energy under Contract No. DE-AC02-05CH11231. This work used resources of the National Energy Research Scientific Computing Center (NERSC). J. Renner (LBNL) acknowledges the support of a US DOE NNSA Stewardship Science Graduate Fellowship under contract no. DE-FC52-08NA28752. This work was also supported by the Ministerio de Economía y Competitividad of Spain under grants CONSOLIDER-Ingenio 2010 CSD2008-0037 (CUP) and FPA2009-13697-C04-04.

\section{References}

[1] E. Majorana, Nuovo Cimento 14 (1937) 171.

[2] J.J. Gómez-Cadenas, J. Martín-Albo, M. Mezzetto, F. Monrabal, M. Sorel, Rivista del Nuovo Cimento 35 (2012) 29.

[3] G. Racah, Nuovo Cimento 14 (1937) 322

[4] J. Schechter, J.W.F. Valle, Physical Review D 25 (1982) 2951.

[5] W. Buchmüller, R. Peccei, T. Yanagida, Annual Review of Nuclear and Particle Science 55 (2005) 311.

[6] A. Pilaftsis, Journal of Physics: Conference Series 171 (2009) 012017.

[7] M. Auger, et al., Physical Review Letters 109 (2012) 032505.

[8] A. Gando, et al., Physical Review C 85 (2012) 045504.

[9] P.M. McCowan, R.C. Barber, Physical Review C 82 (2010) 024603.

[10] U. Fano, Physical Review 72 (1947) 26.

[11] A. Bolotnikov, B. Ramsey, Nuclear Instruments and Methods in Physics Research Section A 396 (1997) 360.

[12] V. Álvarez, et al., Journal of Instrumentation 7 (2012) T06001.

[13] D. Nygren, Nuclear Instruments and Methods in Physics Research Section A 603 (2009) 337.

[14] E. Aprile, A.E. Bolotnikov, A.I. Bolozdynya, T. Doke, Noble Gas Detectors, Wiley VCH, Verlag GmbH \& Co. KGaA, Weinheim, 2006.

[15] V. Álvarez, et al., Journal of Instrumentation 7 (2012) P02010.

[16] A. Bolotnikov, B. Ramsey, IEEE Transactions on Nuclear Science NS-44 (1997) 1006.

[17] G. Mahler, B. Yu, G. Smith, W. Kane, J. Lemley, IEEE Transactions on Nuclear Science NS-45 (1998) 1029.

[18] G. Tepper, J. Losee, Nuclear Instruments and Methods in Physics Research Section A 356 (1995) 339.

[19] C. Levin, J. Germani, J. Markey, Nuclear Instruments and Methods in Physics Research Section A 332 (1993) 206.

[20] R. Kessick, G. Tepper, Nuclear Instruments and Methods in Physics Research Section A 490 (2002) 243.

[21] A. Bolotnikov, A. Bolozdynya, R. DeVito, J. Richards, IEEE Transactions on Nuclear Science NS-51 (2004) 1262.

[22] J. Lacy, A. Athanasiades, N. Shehad, L. Sun, T. Lyons, C. Martin, L. Bu, Cylindrical high pressure xenon spectrometer using scintillation light pulse correction, in: Nuclear Science Symposium Conference Record, 2004 IEEE, vol. 1, pp. 16-20.

[23] S. Kiff, Z. He, G. Tepper, IEEE Transactions on Nuclear Science NS-52 (2005) 2932.

[24] P.N. Luke, Applied Physics Letters 65 (1994) 2884.

[25] A. Bolozdynya, V. Egorov, A. Koutchenkov, G. Safronov, G. Smirnov, S. Medved, V. Morgunov, Nuclear Instruments and Methods in Physics Research Section A 385 (1997) 225.

[26] T.H.V.T. Dias, F.P. Santos, A.D. Stauffer, C.A.N. Conde, Physical Review A 48 (1993) 2887

[27] T.H.V.T. Dias, J.M.F. dos Santos, P.J.B.M. Rachinhas, F.P. Santos, C.A.N. Conde, A.D. Stauffer, Journal of Applied Physics 82 (1997) 2742.

[28] L.P. Ekström, R.B. Firestone, WWW Table of Radioactive Isotopes, database version 2/28/99, 〈http://ie.lbl.gov/toi/index.htm >.

[29] T. Doke, Proceedings of the XeSAT (2005) 92.

[30] R. Platzman, International Journal of Applied Radiation and Isotopes 10 (1961) 116.

[31] F.I.G.M. Borges, J.M.F. dos Santos, T.H.V.T. Dias, F.P. Santos, P.J.B.M. Rachinhas, C.A.N. Conde, Nuclear Instruments and Methods in Physics Research Section A 422 (1999) 321.

[32] C.M.B. Monteiro, L.M.P. Fernandes, J.A.M. Lopes, L.C.C. Coelho, J.F.C.A. Veloso, J.M.F. dos Santos, K. Giboni, E. Aprile, Journal of Instrumentation 2 (2007) P05001. 
[33] C. Silva, J.P. da Cunha, A. Pereira, V. Chepel, M.I. Lopes, V. Solovov, F. Neves, Journal of Applied Physics 107 (2010) 064902.

[34] R. Brun, F. Rademakers, Nuclear Instruments and Methods in Physics Research Section A 389 (1997) 81.

[35] FMWK: a simple framework for HEP data analysis and reconstruction, $\langle$ https://cdcvs.fnal.gov/redmine/projects/nusoft/wiki/FMWK〉.

[36] S.F. Biagi, Nuclear Instruments and Methods in Physics Research Section A 421 (1999) 234.

[37] S. Biagi, MIP, 〈http://consult.cern.ch/writeup/magboltz 〉.
[38] J. Escada, T.H.V.T. Dias, F.P. Santos, P.J.B.M. Rachinhas, C.A.N. Conde A.D. Stauffer, Journal of Instrumentation 6 (2011) P08006.

[39] A. Parsons, T. Edberg, B. Sadoulet, S. Weiss, J. Wilkerson, K. Hurley, R. Lin, G. Smith, IEEE Transactions on Nuclear Science NS-37 (1990) 541.

[40] S.J.C. do Carmo, F.I.G.M. Borges, F.P. Santos, T.H.V.T. Dias, C.A.N. Conde, Journal of Instrumentation 3 (2008) P07004.

[41] L.M.P. Fernandes, E.D.C. Freitas, M. Ball, J.J. Gómez-Cadenas, C.M.B. Monteiro, N. Yahlali, D. Nygren, J.M.F. dos Santos, Journal of Instrumentation 5 (2010) P09006. 